\title{
$F$-actions and parallel-product decomposition of reflexible maps
}

\author{
Alen Orbanić \\ Received: 11 December 2005 / Accepted: 6 March 2007 / \\ Published online: 2 May 2007 \\ (C) Springer Science+Business Media, LLC 2007
}

\begin{abstract}
The parallel product of two rooted maps was introduced by S.E. Wilson in 1994. The main question of this paper is whether for a given reflexible map $M$ one can decompose the map into a parallel product of two reflexible maps. This can be achieved if and only if the monodromy (or the automorphism) group of the map has at least two minimal normal subgroups. All reflexible maps up to 100 edges, which are not parallel-product decomposable, are calculated and presented. For this purpose, all degenerate and slightly-degenerate reflexible maps are classified.

In this paper the theory of $F$-actions is developed including a classification of quotients and parallel-product decomposition. Projections and lifts of automorphisms for quotients and for parallel products are studied. The theory can be immediately applied on rooted maps and rooted hypermaps as they are special cases of $F$-actions.
\end{abstract}

Keywords Rooted map $\cdot F$-Action $\cdot$ Map quotients $\cdot$ Normal quotient $\cdot$ Parallel product $\cdot$ Reflexible map $\cdot$ Parallel-product decomposition

\section{Introduction}

The central problems of reflexible maps are their systematic construction and classification. The most common constructions arise from quotients of extended triangle group [7]. In the classification of reflexible maps, three natural groupings are used, namely by the number of edges [31], by the underlying surface [8] and by the underlying graph [30].

Before the age of fast computers, many authors (Bergau and Garbe [2], Brahana [4], Coxeter and Moser [9], Garbe [11], Sherk [22]) worked on the classification of reflexible and orientably regular maps and managed to classify all such

\footnotetext{
A. Orbanić (ه)

Faculty of Mathematics and Physics, Department of Mathematics, University of Ljubljana,

Jadranska 21, 1000 Ljubljana, Slovenia

e-mail: Alen.Orbanic@fmf.uni-lj.si
} 
maps on surfaces of orientable genus up to 7 and non-orientable genus up to 8 . In the 1970s, Wilson in his Ph.D. thesis [26] calculated most reflexible and orientably regular maps up to 100 edges [31] using a computer and running his Riemann surface algorithm [27]. The recent breakthrough in this field is due to Conder and Dobcsányi [7], who calculated all orientably regular maps on surfaces from genera 3 up to 15 and all non-orientable reflexible maps on surfaces from non-orientable genera 2 up to 30 (Conder-Dobcsányi's census [8]). In 2006 Conder [6] extended the classification to orientable genera up to 100 and non-orientable genera up to 200 .

The purpose of this work is to provide an alternative method for calculation and a shorter description of reflexible maps in terms of certain "primitive" maps from which all other maps can be obtained using some set of operations. The algorithms for performing the operations need to be of relatively low time complexity so the computations of "non-primitive" maps remain simple. It turns out that the appropriate operation is the parallel product introduced by Wilson [29].

The theory in this paper is developed for $F$-actions, a generalization of rooted maps. In this paper it is applied only to reflexible maps, but the same concepts can be used with orientably regular maps, edge-transitive maps $[19,20]$, hypermaps and abstract polytopes.

Overview of main results Usually, a map on a surface is represented by a set of flags and by three involutions, two of which commute, treated as permutations of the flags and intuitively giving instructions for gluing the flags together to form a surface [10, 17, 25]. The group generated by these three involutions acts transitively on the set of flags and is called the monodromy group of the map. The automorphism group of a map is the group of permutations of the flags respecting the action of the monodromy group. A map is reflexible if the automorphism and the monodromy group are regular and isomorphic. A reflexible map is normally parallel-product decomposable if it is a parallel product of two smaller reflexible maps.

The main results of this paper are the following group theoretical characterizations of parallel-product decomposability. In the language of reflexible maps, Theorem 4.5 reads:

Theorem 1.1 A reflexible map is normally parallel-product decomposable if and only if the monodromy group (or the automorphism group) contains at least two different non-trivial minimal normal subgroups.

The theorem is a consequence of the main result of the paper:

Theorem 4.4 (Decomposition theorem) An F-action $M=(f, G, Z$, id) is parallelproduct decomposable if and only if there exist two different subgroups $K_{1}, K_{2} \leq G$, such that $G_{\underline{\mathrm{id}}} \lesseqgtr K_{i} \lesseqgtr G, i=1,2$, and $G_{\underline{\mathrm{id}}}=K_{1} \cap K_{2}$. Furthermore, $M$ is normally parallel-product decomposable if and only if there exist two different non-trivial normal subgroups $H_{1}, H_{2} \triangleleft G$ acting non-transitively on $Z$ and $G_{\text {id }} H_{1} \cap G_{\underline{\text { id }}} H_{2}=G_{\text {id }}$. Also, $M$ is normally parallel-product decomposable if and only if it is strictly parallel-product decomposable. 
Among all the groups up to order 1000 , only $0.1 \%$ of groups have unique minimal normal subgroup (the actual ratio is 12860/11758814). According to Theorem 4.5, only these groups may support reflexible maps which are "primitive".

Paper layout The sections of this paper are organized as follows.

Section 2 provides us with basic definitions for $F$-actions and establishes the algebraic machinery necessary to discuss them in a manner similar to the article about Cayley maps [21].

In Sect. 3 we establish the correspondence between $F$-actions and the lattice of subgroups of the finitely presented group $F$ in a manner similar to [5]. The correspondence helps us to analyze and characterize $F$-action morphisms. We introduce $K$-quotients and normal quotients. In Theorem 3.5 we prove that any $F$-action morphism arises from some $K$-quotient. A normal quotient has the special property that all automorphisms project, and it is used in the normal parallel-product decomposition in the next section.

Section 4 contains the main result and some propositions describing properties of the parallel product, mainly focusing on lifts of automorphisms. If factors have high symmetry, then their parallel product is also highly symmetric. Using the correspondence from the previous section we are able to characterize parallel-product decomposability of an $F$-action through the subgroup lattice of its monodromy group.

Section 5 classifies all degenerate and slightly degenerate reflexible maps. These are basically the maps containing vertices of valence less than 3 or some kind of degeneracy of edges, such as loops or semi-edges. All the maps obtained from those by triality are also included. These degeneracies arise naturally in quotients. All non-degenerate normally parallel-product indecomposable reflexible maps up to 100 edges are listed.

In Sect. 6 decomposability of degenerate and slightly degenerate reflexible maps is characterized and all parallel-product indecomposable reflexible maps up to 100 edges are listed.

\section{Definitions}

A right action of a group $G$ on a finite set $Z$ is an operation $\cdot: Z \times G \rightarrow Z$, such that $z \cdot 1=z$ and $z \cdot(g h)=(z \cdot g) \cdot h$, for every $z \in Z$ and $g, h \in G$. We denote the action by a pair $(Z, G)$. Denote by $\operatorname{Sym}_{\mathrm{R}}(Z)$ the symmetric group on the set $Z$, where the bijections (permutations) are composed from the left to the right and naturally act on $Z$ from the right. For $g \in G$, a mapping $\pi_{g}: Z \rightarrow Z$, $\pi_{g}: x \mapsto x \cdot g$ is a bijection on $Z$ and therefore an element of $\operatorname{Sym}_{\mathrm{R}}(Z)$. The mapping $\chi: G \rightarrow \operatorname{Sym}_{\mathrm{R}}(Z), \chi: g \mapsto \pi_{g}$ is a group homomorphism and is called the action homomorphism. The image $\chi(G) \leq \operatorname{Sym}_{\mathrm{R}}(Z)$ is called the image of the action and $\operatorname{ker} \chi$ is called the kernel of the action. The stabilizer of an element $z \in Z$ is the group $G_{z}=\{g \in G \mid z \cdot g=z\}$. The kernel of the action is exactly the intersection of all the stabilizers. The action is semi-regular if all $G_{z}$ are trivial, faithful if the kernel is trivial, transitive if for any two $z, z^{\prime} \in Z$ there exists $g \in G$, such that $z \cdot g=z^{\prime}$. Denote by $\operatorname{Core}_{G}(K)=\bigcap_{g \in G} K^{g}$, the core of a subgroup $K$ in $G$, which is the intersection of all the conjugates of $K$ and also the maximal normal subgroup 
in $G$ contained in $K$. All stabilizers of a transitive action $(Z, G)$ are conjugate and the kernel equals $\operatorname{Core}_{G}\left(G_{z}\right)$, for any $z \in Z$. A transitive semi-regular action is regular.

An action epimorphism of two right actions $(Z, G)$ and $(W, H)$ is a pair $(\phi, \psi)$, where $\phi: Z \rightarrow W$ is an onto mapping, $\psi: G \rightarrow H$ is a group epimorphism, and for every $z \in Z$ and $g \in G$ we have $\phi(z \cdot g)=\phi(z) \cdot \psi(g)$. If both $\phi$ and $\psi$ are one-to-one, then $(\phi, \psi)$ is an action isomorphism.

In a similar manner, but changing the sides, a left action is defined and denoted by $(G, Z)$. In the case of a group $G$ acting on itself, the notation $(G, G)$ is confusing; therefore the nature of the action (left or right) is explained in the context. Mostly, right actions will be used in the paper. Left actions will occur only when automorphism groups are involved.

A rooted transitive action $(R T A)$ is a triple $(Z, G, \underline{\mathrm{id}})$, where $(Z, G)$ is a transitive action and $\underline{\mathrm{id}} \in Z$ is the distinguished element called the root. An RTA morphism is an action epimorphism which maps a root to a root.

Let $F=\left\langle a_{1}, \ldots, a_{k} \mid R_{1}=\cdots=R_{n}=1\right\rangle$ be a finitely presented group with generators $\left\{a_{i}\right\}_{i=1}^{k}$ and relations $\left\{R_{j}\right\}_{j=1}^{n}$. An $F$-group is a pair $(f, G)$, where $f: F \rightarrow G$ is a group epimorphism. An $F$-group morphism of two $F$-groups $A_{i}=\left(f_{i}, G_{i}\right)$, $i=1,2$, is a group epimorphism $\psi: G_{1} \rightarrow G_{2}$, such that $\psi \circ f_{1}=f_{2}$. Note that this implies that if a morphism exists, then it is unique. If $\psi$ is an isomorphism, we denote this by $A_{1} \simeq A_{2}$. An $F$-group should be viewed as a group with a specified subset of (labelled) generators. Also it can be viewed as a quotient of $F$, as its finite presentation is just the presentation of $F$ with additional relations.

A (finite) $F$-action is a 4-tuple $M=(f, G, Z$, $\underline{\mathrm{id}})=\left(f_{M}, G_{M}, Z_{M}, \underline{\mathrm{id}}_{M}\right)$, where $Z$ is a set of flags, $(Z, F, \underline{\text { id })}$ is an RTA, $(Z, G, \underline{\text { id })}$ is a faithful RTA, (Id, $f)$ : $(Z, F, \underline{\mathrm{id}}) \rightarrow(Z, G, \underline{\mathrm{id}})$ is an RTA morphism (here Id denotes the identity mapping) and $(f, G)$ is an $F$-group. Let $\chi_{M}$ denote the action homomorphism $\chi_{M}: G \rightarrow$ $\operatorname{Sym}_{\mathrm{R}}(Z)$. Then $\operatorname{Mon}(M)=\left(\chi_{M} \circ f, \chi_{M}(G)\right)$ is an $F$-group called the monodromy group. It is considered as a permutation group with labelled generators and as such a particularly convenient representation of an $F$-action when doing computer calculations. Define $\mathrm{S}_{F}(M)=F_{\underline{\mathrm{id}}}=f^{-1}\left(G_{\underline{\mathrm{id}}}\right)$, the stabilizer of id in $F$.

Example 2.1 A finite map on a closed compact surface $S$ is an embedding of a finite connected graph $X$ on $S$, where $S \backslash X$ consists of connected parts homeomorphic to disks (faces). According to $[10,17,25]$, such a map can be combinatorially represented by a finite set of flags $Z$ and three fixed-point-free involutions $T, L, R \in \operatorname{Sym}_{\mathrm{R}}(Z)$, where $T$ and $L$ commute and $T L$ is also fixed-point-free. The involutions act on the set of flags and generate the monodromy group. Imagine the flags as triangles with the sides labelled by $T, L$ and $R$ and glue two triangles $a$ and $b$ along the side labelled by $T$, if $a T=b$, and do similarly for the labels $L$ and $R$. The conditions on the involutions imply that the surface obtained by gluing is a compact closed surface and the sides labelled by $T$ define an embedding of a graph. If we do not insist on the involutions being fixed-point free, we obtain algebraic objects called holey maps as defined in [1]. If we additionally root them, we get $\mathcal{M}$-actions for $\mathcal{M}=\left\langle T, L, R \mid T^{2}=L^{2}=R^{2}=(T L)^{2}=1\right\rangle$, where the involutions correspond to labelled generators of monodromy groups.

Example 2.2 An orientable map can be described in terms of half-edges called darts, a permutation $R$ on darts, which encodes local rotations in vertices, and an involu- 
tion $L$, where for a dart $z, z \cdot L$ denotes the other half of the edge (dart). By additionally rooting such a map, we get an $\mathcal{O}$-action for $\mathcal{O}=\left\langle R, L \mid L^{2}=1\right\rangle$.

Example 2.3 A hypermap can be described in terms of three involutions acting on the set of flags. Allowing certain degeneracies and rooting a hypermap, it can be considered as an $\mathcal{H}$-action for $\mathcal{H}=\left\langle r_{0}, r_{1}, r_{2} \mid r_{0}^{2}=r_{1}^{2}=r_{2}^{2}=1\right\rangle$ (see [5]).

Example 2.4 A $k$-constellation is a sequence of permutations $\left[\phi_{1}, \ldots, \phi_{k}\right]$ in $S_{n}$, where $\prod_{i=1}^{k} \phi_{i}=1$ and the permutations admit certain relations (see [32] for examples). Constellations are exactly generator sequences of monodromy groups of certain $F$-actions.

As we can see, an $F$-action is nothing but a transitive action of the finitely presented group $F$ on a finite set together with its faithful presentation (in practice it is usually a permutation representation).

Let $M$ and $N$ be $F$-actions. An $F$-action morphism is a pair $(\phi, \psi)$, that is an RTA morphism $(\phi, \psi):\left(Z_{M}, G_{M}, \underline{\mathrm{id}}_{M}\right) \rightarrow\left(Z_{N}, G_{N}, \underline{\mathrm{id}}_{N}\right)$ and $\psi:\left(f_{M}, G_{M}\right) \rightarrow$ $\left(f_{N}, G_{N}\right)$ is an $F$-group morphism. A map morphism is called strict provided that ker $\psi$ is non-trivial. If $\phi, \psi$ are one-to-one, we have an $F$-action isomorphism, and we write $M \simeq N$. Note that $\operatorname{Mon}(M) \simeq \operatorname{Mon}(N)$ if and only $\left(f_{M}, Z_{M}\right) \simeq\left(f_{N}, Z_{N}\right)$. While $M \simeq N$ implies $\operatorname{Mon}(M) \simeq \operatorname{Mon}(N)$, the converse is far from being true. If there is a map morphism $(\phi, \psi): M \rightarrow N$ between two $F$-actions, then it is unique: let $x=\underline{\mathrm{id}}_{M} \cdot f_{M}(v), v \in F$; then $\phi\left(x \cdot f_{M}(w)\right)=\phi\left(\underline{\mathrm{id}}_{M} \cdot f_{M}(v w)\right)=$ $\underline{\mathrm{id}}_{N} \cdot \psi\left(f_{M}(v w)\right)=\underline{\mathrm{id}}_{N} \cdot f_{N}(v w)$, for any $w \in F$. The existence of a morphism $M \rightarrow N$ is denoted by $N \leq M$.

An automorphism of an $F$-action $M$ is an action isomorphism $(\phi, \mathrm{Id}):\left(Z_{M}, G_{M}\right)$ $\rightarrow\left(Z_{M}, G_{M}\right)$, where Id denotes the identity mapping. Note that contrary to an $F$-action morphism, here the condition $\phi\left(\underline{\mathrm{id}}_{M}\right)=\underline{\mathrm{id}}_{N}$ is omitted and therefore in general, an $F$-action automorphism is not an $F$-action morphism in the categorical sense. The group of all automorphisms is denoted by $\operatorname{Aut}(M)$. Since an automorphism is completely determined by the image of a single flag, the action of $\operatorname{Aut}(M)$ on $Z$ is semi-regular. We write automorphisms on the left, so that the image of $z$ un$\operatorname{der} p$ is $p(z)$. Thus the action of $\operatorname{Aut}(M)$ is a left action. An $F$-action $M$ is regular if $\operatorname{Aut}(M)$ acts regularly on flags. The symbol $\alpha_{w}$ will denote the automorphism in $\operatorname{Aut}(M)$ (if it exists) that takes $\underline{\mathrm{id}}_{M}$ to $\underline{\mathrm{id}}_{M} \cdot w$, where $w \in F$. If $p \in \operatorname{Aut}(M)$ is such an automorphism, we will denote this by $p \equiv \alpha_{w} \in \operatorname{Aut}(M)$ and say that $M$ contains $\alpha_{w}$.

The flag graph of an $F$-action $M$ is the directed multi-graph with labelled edges, where the set of the vertices is $Z_{M}$ and for each $z \in Z_{M}$ and each $a \in\left\{a_{i}^{ \pm 1}\right\}_{i=1}^{k}$, there is a directed edge from $z$ to $z \cdot f_{M}(a)$ with the label $a$. Note that in a situation where $z \cdot a=z$ we have a loop, if the order of $a$ in $F$ is greater than 2, or a semi-edge otherwise.

Denote by $F^{+}$the subgroup of even length words in $F$. Depending on the relations in $F, F^{+}$can be either equal to $F$ or a subgroup of index 2 . In the latter case, we call an $F$-action $M$ orientable if and only if $F^{+}$has exactly two orbits on $Z_{M}$. For an interpretation of orientability see Example 2.5 below.

If a root flag $\underline{\mathrm{id}}_{M}$ of an $F$-action $M$ is changed to the flag $\underline{\mathrm{id}}_{M} \cdot w, w \in F$, a rerooted $F$-action $R_{w}(M)$ is obtained. Note that $\mathrm{S}_{F}\left(R_{w}(M)\right)=w^{-1} \mathrm{~S}_{F}(M) w$. 
Let $d \in \operatorname{Aut}(F)$ and $M=(f, G, Z$, id). Then $d$ induces an $F$-action operation $O_{d}(M)=(f \circ d, G, Z$, id $)$. If $d$ is an inner automorphism, say conjugation by $w$, then it is quite easy to see that $O_{d}(M) \simeq R_{w^{-1}}(M)$.

For a subgroup $K \leq F$ and an $F$-action $M$, the orbits of $K$ acting on $Z_{M}$ are called $K$-orbits and are blocks of imprimitivity for the left action of the automorphism group.

Let $p: M \rightarrow N$ be a morphism of $F$-actions and $f \in \operatorname{Aut}(M)$. If there exists $f^{\prime} \in$ $\operatorname{Aut}(N)$, such that $p \circ f=f^{\prime} \circ p$, then we say that $f$ projects (along $p$ ). On the other hand, if there is $f^{\prime} \in \operatorname{Aut}(N)$ and there exists $f \in \operatorname{Aut}(M)$, such that $p \circ f=f^{\prime} \circ p$, we say that $f$ lifts (with $p$ ). Note that for $w \in F$, if $\alpha_{w} \in \operatorname{Aut}(M)$ projects, it projects to $\alpha_{w} \in \operatorname{Aut}(N)$.

Example 2.5 For holey maps ( $\mathcal{M}$-actions), combinatorial edges, vertices, faces and Petrie circuits are the $\langle T, L\rangle-,\langle T, R\rangle-,\langle L, R\rangle$ - and $\langle L T, R\rangle$-orbits, respectively. Consider the automorphisms $\mathbf{d}, \mathbf{p} \in \operatorname{Aut}(\mathcal{M})$, defined by the assignments $\mathbf{d}: T \mapsto L$, $L \mapsto T, R \mapsto R$ and $\mathbf{p}: T \mapsto T, L \mapsto T L, R \mapsto R$. They induce the well known map operations, the dual $\mathrm{D}(M)=O_{\mathbf{d}}(M)$ and the Petrie dual $\mathrm{P}(M)=O_{\mathbf{p}}(M)$. The orbits of the left action of the group $\langle\mathrm{D}, \mathrm{P}\rangle$ on the set of all holey maps are called the triality classes and since $\langle\mathrm{D}, \mathrm{P}\rangle \simeq S_{3}$ (according to [15]), a triality class can contain 1,2,3 or 6 holey maps. Regular holey maps on surfaces are called reflexible maps. Note that here $\mathcal{M}^{+}=\langle R T, L R\rangle$. A holey map $M$ is embedded on a orientable closed compact surface $S$ if and only $M$ is orientable as an $\mathcal{M}$-action.

Example 2.6 In an orientable map (see Example 2.2) vertices can be considered as $\langle R\rangle$-orbits, edges as $\langle L\rangle$-orbits and faces as $\langle R L\rangle$-orbits. For an automorphism $g$ of $\mathcal{O}=\left\langle R, L \mid L^{2}=1\right\rangle$ taking $R$ to $R^{-1}$ and keeping $L$ fixed we get an operation $O_{g}$ returning a mirror image of an orientable map. Note that the map is chiral if and only if $M$ is not isomorphic to $O_{g}(M)$ as an $\mathcal{O}$-action.

Example 2.7 Automorphism groups of edge-transitive maps (holey maps; automorphism group transitive on $\langle T, L\rangle$-orbits) admitting type $\mathcal{T}$ are quotients of certain finitely presented groups $F_{\mathcal{T}}$ (see [23]; $F_{\mathcal{T}}$ is called a partial presentation). The following paragraph describes how edge-transitive maps admitting type $\mathcal{T}$ can be viewed as regular $F_{\mathcal{T}}$-maps.

For the type $2 e x^{P}, F_{2 e x^{P}}=\left\langle\sigma_{x_{1}}, \varphi \mid \varphi^{2}=1\right\rangle$, where $\sigma_{x_{1}} \equiv \alpha_{R T}$ and $\varphi \equiv \alpha_{T L}$. Consider the action of $\operatorname{Aut}(M)$ on $\langle T\rangle$-orbits, i.e. darts, which happens to be regular. As we will see later (Theorem 3.6), the mapping $\Phi: F_{2 e x} P \rightarrow\langle R T, T L\rangle$ defined by $\sigma_{x_{1}} \mapsto R T, \varphi \mapsto T L$, is a group isomorphism. Define $\mathbf{R}=R T$ and $\mathbf{L}=T L$. Then the finite presentation of $\langle\mathbf{R}, \mathbf{L}\rangle$ is $\left\langle\mathbf{R}, \mathbf{L} \mid \mathbf{L}^{2}=1\right\rangle$. The maps admitting type $2 \operatorname{ex}^{P}$ are exactly orientably regular maps (see Example 2.2).

Similar approach leads us to the conclusion that edge-transitive maps admitting type $\mathcal{T}$ are exactly regular $F_{\mathcal{T}}$-maps.

Using this approach and the presentations from [23], edge-transitive maps admitting types $2,2^{*}$ and $2^{P}$ can be considered as regular $F$-actions, where $F=$ $\left\langle a, b, c \mid a^{2}=b^{2}=c^{2}=1\right\rangle$. Therefore, all three types are algebraically equivalent to regular hypermaps (or $\mathcal{H}$-actions, see Example 2.3). 


\section{Quotients of $\boldsymbol{F}$-actions}

This section extends the results by Wilson [29] and by Breda d'Azevedo and Nedela [5]. Different parts of similar topics were discussed also in [18] (for orientably regular maps), [12,13] (for abstract polytopes) and [21] (for Cayley maps). Wilson was the first to introduce the parallel product, while Breda d'Azevedo and Nedela discovered the connection between regular $\mathcal{H}$-actions and the normal subgroup lattice of $\mathcal{H}$ (hypermaps). Their idea is used to show the connection between $F$-actions and the subgroup lattice of $F$ which is then used to prove some interesting results. The connection is established through the stabilizer of the root flag.

If $(Z, G)$ is a transitive action with the kernel $H$, then the induced action $(Z, G / H)$ is faithful. For example, if $K \leq G$ are groups, then $(G / K, G, K)$ is an RTA and if $H=\operatorname{Core}_{G}(K)$, then $(G / K, G / H, K)$ is a faithful RTA explicitly defined by $K w \cdot H v=K w v$, for $w, v \in G$. For $K \leq F$, define $\mathrm{M}_{\mathrm{F}}(K)=$ $\left(q, F / \operatorname{Core}_{F}(K), F / K, K\right)$, where $q: F \rightarrow F / \operatorname{Core}_{F}(K)$ is the natural epimorphism. Obviously, $\mathrm{M}_{\mathrm{F}}(K)$ is an $F$-action. Note that for $K \leq F, \mathrm{~S}_{F}\left(\mathrm{M}_{\mathrm{F}}(K)\right)=K$.

Proposition 3.1 Let $M$ be an F-action. Then $M \simeq \mathrm{M}_{\mathrm{F}}\left(\mathrm{S}_{F}(M)\right)$.

Proof Let $M=(f, G, Z, \underline{\mathrm{id}}), K=\mathrm{S}_{F}(M), H=\operatorname{Core}_{F}(K), q: F \rightarrow F / H$ be the natural epimorphism and $\mathrm{M}_{\mathrm{F}}\left(\mathrm{S}_{F}(M)\right)=(q, F / H, F / K, K)$. Let $w \in F, z \in Z$, such that $z=\underline{\mathrm{id}} \cdot w$ and define $\phi(z)=K w$. Since $\underline{\mathrm{id}} \cdot w=\underline{\mathrm{id}} \cdot v$, if and only if $w v^{-1} \in K$, $\phi$ is well defined and one-to-one. Obviously, $\phi$ is onto and $\phi(\underline{\mathrm{id}})=K$. As $(Z, G)$ is faithful, $\operatorname{ker} f=H$. Since $f$ is an epimorphism with kernel $H$ and image $G$, there exists an isomorphism $\psi: G \rightarrow F / H$, such that $\psi \circ f=q$.

Let $z \in Z, g \in G$ and $w, v \in F$ be such that $f(w)=g$ and $\underline{\mathrm{id}} \cdot v=z$. Then $\phi(z \cdot g)=\phi(z \cdot f(w))=\phi((\underline{\mathrm{id}} \cdot v) \cdot w)=\phi(\underline{\mathrm{id}} \cdot(v w))=K v w$, while $\phi(z) \cdot \psi(g)$ $=\phi(z) \cdot \psi(f(w))=K v \cdot q(w)=K v \cdot H w=K v w$. Hence $(\phi, \psi)$ is the isomorphism.

Proposition 3.2 Let $K_{1}, K_{2} \leq F$ be subgroups. Then $K_{1} \leq K_{2}$ if and only if there exists an $F$-action morphism $(\phi, \psi): \mathrm{M}_{\mathrm{F}}\left(K_{1}\right) \rightarrow \mathrm{M}_{\mathrm{F}}\left(K_{2}\right)$.

Proof For $i=1,2$, let $H_{i}=\operatorname{Core}_{F}\left(K_{i}\right), q_{i}: F \rightarrow F / H_{i}$ be the natural epimorphisms and $\mathrm{M}_{\mathrm{F}}\left(K_{i}\right)=\left(q_{i}, F / H_{i}, F / K_{i}, K_{i}\right)$.

If $K_{1} \leq K_{2}$, then $H_{1} \leq H_{2}$ and the mappings $\psi: F / H_{1} \rightarrow F / H_{2}, \psi: H_{1} w \mapsto$ $H_{2} w$ and $\phi: F / K_{1} \rightarrow F / K_{2}, \phi: K_{1} w \mapsto K_{2} w$, for any $w \in F$, are well defined. Also, $\psi$ is an epimorphism and $\psi \circ q_{1}=q_{2}$. For every $w, v \in F$, it follows that $\phi\left(K_{1} w \cdot H_{1} v\right)=\phi\left(K_{1} w v\right)=K_{2} w v$ and $\phi\left(K_{1} w\right) \cdot \psi\left(H_{1} v\right)=K_{2} w \cdot H_{2} v=K_{2} w v$. Since $\phi\left(K_{1}\right)=K_{2}$ and $\phi$ and $\psi$ are onto, $(\phi, \psi): \mathrm{M}_{\mathrm{F}}\left(K_{1}\right) \rightarrow \mathrm{M}_{\mathrm{F}}\left(K_{2}\right)$ is an $F$-action morphism.

On the other hand, let $(\phi, \psi): \mathrm{M}_{\mathrm{F}}\left(K_{1}\right) \rightarrow \mathrm{M}_{\mathrm{F}}\left(K_{2}\right)$ be an $F$-action morphism. Then $q_{2}=\psi \circ q_{1}$ and $\phi\left(K_{1}\right)=K_{2}$. Let $x \in K_{1}$. Then $q_{1}(x) \in K_{1} / H_{1}=\left(F / H_{1}\right)_{K_{1}}$. Since $(\phi, \psi)$ is an $F$-action morphism, it is true that $\psi\left(\left(F / H_{1}\right)_{K_{1}}\right) \leq\left(F / H_{2}\right)_{K_{2}}=$ $K_{2} / H_{2}$. Therefore $\left(\psi \circ q_{1}\right)(x)=q_{2}(x) \in K_{2} / H_{2}$, implying that $x \in K_{2}$. Hence, $K_{1} \leq K_{2}$.

From the last two propositions the next corollary immediately follows. 
Corollary 3.3 Let $M$ and $N$ be $F$-actions. Then there exists an $F$-action morphism $(\phi, \psi): M \rightarrow N$ if and only if $\mathrm{S}_{F}(N) \leq \mathrm{S}_{F}(M)$. Therefore, $M \simeq N$ if and only if $\mathrm{S}_{F}(M)=\mathrm{S}_{F}(N)$.

Recall the elementary theorem known as the fourth isomorphism theorem for groups (the correspondence theorem).

Theorem 3.4 Let $G, G^{\prime}$ be groups and $f: G \rightarrow G^{\prime}$ epimorphism. Let $\mathcal{A}=\{K$ : $\operatorname{ker} f \leq K \leq G\}$ and $\mathcal{B}=\left\{K^{\prime}: K^{\prime} \leq G^{\prime}\right\}$. Then the mapping $\Sigma: \mathcal{A} \rightarrow \mathcal{B}$ defined by $\Sigma: K \mapsto f(K)$ is a bijection. Under this bijection normal subgroups correspond to normal subgroups. For any two groups $K, H \in \mathcal{A}$, it follows $\Sigma(K \cap H)=\Sigma(K) \cap$ $\Sigma(H), \Sigma(\langle K, H\rangle)=\langle\Sigma(K), \Sigma(H)\rangle$ and if $K \leq H$, then $\Sigma(K) \leq \Sigma(H)$.

Note that the sets of groups $\mathcal{A}$ and $\mathcal{B}$ are actually lattice intervals in lattices of subgroups of $G$ and $G^{\prime}$, respectively. The theorem is also called the lattice theorem for groups, since it basically says that $f$ induces a lattice isomorphism between the two lattice intervals with a special property of mapping normal subgroups to normal subgroups.

For an $F$-action $M=(f, G, Z$, id $)$ and a subgroup $K \leq G$, where $G_{\underline{\text { id }}} \leq K$, define $M / K=(q \circ f, G / H, G / K, K)$, where $H=\operatorname{Core}_{G}(K)$ and $q: G \rightarrow G / H$ is the natural epimorphism. The right action of $G / H$ on $G / K$ is faithful and $M / K$ is an $F$-action called the $K$-quotient of the $F$-action $M$. A $K$-quotient of $M$ is strict if Core $_{G}(K)$ is not trivial.

Theorem 3.5 Let $M$ and $N$ be $F$-actions, such that there exists an $F$-action morphism $(\phi, \psi): M \rightarrow N$. Let $K=\psi^{-1}\left(\left(G_{N}\right)_{\underline{\mathrm{id}}_{N}}\right) \leq G_{M}$. Then $\left(G_{M}\right)_{\underline{\mathrm{id}}_{M}} \leq K$ and $M / K \simeq N$.

For any two $N, N^{\prime} \leq M$, where $N \simeq M / K, N^{\prime} \simeq M / K^{\prime}$ for some $K, K^{\prime} \leq G_{M}$, it follows $N \leq N^{\prime}$ if and only if $K^{\prime} \leq K$. Also, $N \simeq N^{\prime}$ if and only if $K=K^{\prime}$.

Proof Since $\left(\mathrm{Id}, f_{M}\right):\left(Z_{M}, F, \underline{\mathrm{id}}_{M}\right) \rightarrow\left(Z_{M}, G_{M}, \underline{\mathrm{id}}_{M}\right)$ is an RTA morphism and Id is a bijection, it follows $f_{M}\left(F_{\underline{\mathrm{id}}}\right)=\left(G_{M}\right)_{\underline{\mathrm{id}}}$ and $f_{M}^{-1}\left(\left(G_{M}\right)_{\underline{\mathrm{id}}_{M}}\right)=F_{\underline{\mathrm{id}}}$. As $N \leq M$, it follows $X=\mathrm{S}_{F}(N) \geq \mathrm{S}_{F}(M)=F_{\underline{\mathrm{id}}_{M}}$, by Corollary 3.3 . Let $K=f_{M}(X)$. Since $f_{M}$ is an epimorphism and $\operatorname{ker} f_{M} \leq F_{\text {id }_{M}} \leq X$, it is true that $f_{M}^{-1}(K)=X$ and $\left(G_{M}\right)_{\underline{\mathrm{id}}_{M}} \leq K$, by Theorem 3.4. But for $M / K=\left(q \circ f_{M}, G_{M} / H, G_{M} / K, K\right)$, where $H=\operatorname{Core}_{G_{M}}(K)$ and $q: G_{M} \rightarrow G_{M} / H$ is the natural epimorphism, it follows $\left(G_{M} / H\right)_{K}=K / H$, and thus $\mathrm{S}_{F}(M / K)=\left(q \circ f_{M}\right)^{-1}(K / H)=f_{M}^{-1}(K)$ $=X$. By Corollary 3.3, $M / K \simeq N$. As $f_{M}$ is an epimorphism, $K=f_{M}(X)=$ $f_{M}\left(f_{N}^{-1}\left(\left(G_{N}\right)_{\underline{\mathrm{id}}_{N}}\right)\right)=f_{M}\left(f_{M}^{-1}\left(\psi^{-1}\left(\left(G_{N}\right)_{\underline{\mathrm{id}}_{N}}\right)\right)\right)=\psi^{-1}\left(\left(G_{N}\right)_{\underline{\mathrm{id}}_{N}}\right)$.

Let $N, N^{\prime} \leq M$. Then $N \leq N^{\prime}$ if and only if $\mathrm{S}_{F}(N) \geq \mathrm{S}_{F}\left(N^{\prime}\right)$, if and only if $K=f_{M}\left(\mathrm{~S}_{F}(N)\right) \geq f_{M}\left(\mathrm{~S}_{F}\left(N^{\prime}\right)\right)=K^{\prime}$, by Theorem 3.4 and Corollary 3.3. Therefore, $K=K^{\prime}$ if and only if $\mathrm{S}_{F}(N)=\mathrm{S}_{F}\left(N^{\prime}\right)$ if and only if $N \simeq N^{\prime}$.

The role of Theorem 3.5 for $F$-actions is similar to the role of the first isomorphism theorem for groups. From a computational point of view, it enables us to calculate all the quotients of an $F$-action from the monodromy group. 
Theorem 3.6 Let $M=\left(f, G, Z\right.$, id) and $\mathrm{S}_{F}(M)=K$. For $w \in F, \alpha_{w} \in \operatorname{Aut}(M)$ if and only if $w \in N_{F}(K)$, where $N_{F}(K)$ is, as usual, the normalizer of $K$ in $F$. Furthermore, $\operatorname{Aut}(M) \simeq N_{F}(K) / K$.

Proof Let $H=\operatorname{Core}_{F}(K)$ and $L=\mathrm{M}_{\mathrm{F}}\left(\mathrm{S}_{F}(M)\right)=(q, F / H, F / K, K)$. As $L \simeq M$ by Proposition 3.1, $\alpha_{w} \in \operatorname{Aut}(L)$ if and only if $\alpha_{w} \in \operatorname{Aut}(M)$.

Let $\alpha_{w} \in \operatorname{Aut}(L)$ and $x \in w^{-1} K w$. Then $x=w^{-1} k w$, for some $k \in K$, and $\alpha_{w}\left(K w^{-1}\right)=\alpha_{w}(K) \cdot w^{-1}=K w w^{-1}=K$. Also $K x=\alpha_{w}\left(K w^{-1}\right) \cdot x=$ $\alpha_{w}\left(K w^{-1} w k w^{-1}\right)=\alpha\left(K w^{-1}\right)=K$ and $x \in K$. Hence, $w K w^{-1}=K$ and $w \in$ $N_{F}(K)$.

If $w \in N_{F}(K)$, then $w K w^{-1}=K$ and $w K=K w$. Let $\phi: F / K \rightarrow F / K$ be defined by $\phi: K x \mapsto w K x=K w x$, for any $x \in F$. Obviously, $\phi$ is well defined and a bijection. Then for any $v \in F, \phi(K x) \cdot v=K w x v$ and $\phi(K x \cdot v)=\phi(K x v)=$ $K w x v$. Since $\phi(K)=K w,(\phi, \operatorname{Id}) \equiv \alpha_{w} \in \operatorname{Aut}(L)$.

Define a mapping $\Phi: \operatorname{Aut}(L) \rightarrow N_{F}(K) / K$, where $\Phi: \alpha_{w} \mapsto K w$. Since $\alpha_{w}$ and $\alpha_{v}$ represent the same automorphism in $\operatorname{Aut}(L)$ if and only if $w v^{-1} \in K, \Phi$ is well defined and one-to-one. Also, $\Phi\left(\alpha_{w v}\right)=K w v=K w K v=\Phi\left(\alpha_{w}\right) \Phi\left(\alpha_{v}\right)$, since $K \triangleleft N_{F}(K)$. By the above discussion, $\Phi$ is onto.

Note that the Theorem 3.6 appears in similar forms in several papers which deal with different types of $F$-actions (Cayley maps [21], hypermaps [5], abstract polytopes [13]).

Two corollaries immediately follow.

Corollary 3.7 An F-action $M$ is regular if and only if $\mathrm{S}_{F}(M) \triangleleft F$. M is regular if and only if $\operatorname{Aut}(M)$ and $\operatorname{Mon}(M)$ are isomorphic as abstract groups.

Proof The first part follows directly from Theorem 3.6. If $M$ is regular, let $H=$ $\mathrm{S}_{F}(M) \triangleleft F$ and $q: F \rightarrow F / H$ the natural epimorphism. Then $\mathrm{M}_{\mathrm{F}}\left(\mathrm{S}_{F}(M)\right)=$ $(q, F / H, F / H, K), \operatorname{Mon}(M) \simeq(q, F / H)$ (as $F$-groups) and $\operatorname{Aut}(M) \simeq F / H$, by Theorem 3.6. If $M$ is not regular, then $|\operatorname{Aut}(M)|<\left|Z_{M}\right| \leq|\operatorname{Mon}(M)|$, since $\operatorname{Mon}(M)$ is transitive.

Corollary 3.8 Let $M, N$ be $F$-actions and $p: M \rightarrow N$ be an $F$-action morphism. Then $\operatorname{Aut}(M)$ projects if and only if $N_{F}\left(\mathrm{~S}_{F}(M)\right) \leq N_{F}\left(\mathrm{~S}_{F}(N)\right)$.

Proof Note that $\alpha_{w} \in \operatorname{Aut}(M)$ projects if and only if it projects to $\alpha_{w} \in \operatorname{Aut}(N)$.

Let $M=(f, G, Z$, id) and let $H \triangleleft G$. Consider the RTA morphism (Id, $f)$ : $(Z, F, \underline{\mathrm{id}}) \rightarrow(Z, G, \underline{\mathrm{id}})$ of $M$. By Theorem 3.4, $\mathrm{S}_{F}\left(M / G_{\underline{\mathrm{id}}} H\right)=f^{-1}\left(G_{\underline{\mathrm{id}}} H\right)=$ $f^{-1}\left(G_{\underline{\text { id }}}\right) \cdot f^{-1}(H)=\mathrm{S}_{F}(M) f^{-1}(H)$. Since $f^{-1}(H) \triangleleft F$, it follows $N_{F}\left(\overline{\mathrm{S}}_{F}(M)\right)$ $\leq N_{F}\left(\overline{\mathrm{S}}_{F}\left(M / G_{\text {id }} H\right)\right)$. Therefore $\operatorname{Aut}(M)$ projects, by Corollary 3.8. A $K$-quotient, where $K=G_{\underline{\mathrm{id}}} \bar{H}, H \triangleleft G$ is called a normal quotient and is denoted by $M \triangle H$. The following proposition summarizes the discussion.

Proposition 3.9 Let $M$ be an F-action and $p: M \rightarrow M \triangle H$ be the F-action morphism onto the normal quotient. Then $\operatorname{Aut}(M)$ projects along $p$. 
The reader can easily verify the following construction. Let $M$ be an $F$-action and $H \triangleleft G_{M}$. Let $Z_{M} / H$ denote the set of the orbits of $H$ acting on $Z_{M}$. Then the induced action $\left(Z_{M} / H, G_{M}\right)$ is transitive. If $\left[\underline{\mathrm{id}}_{M}\right.$ ] denotes the orbit in $Z_{M} / H$ containing $\underline{\mathrm{id}}_{M}$ and $q: G_{M} \rightarrow G_{M} / H$ is the natural epimorphism, then the stabilizer of $\left[\underline{\mathrm{id}}_{M}\right]$ is $G_{\mathrm{id}} H$, and $\left.\left(q \circ f_{M}, G_{M} / \operatorname{Core}_{G}\left(G_{\text {id }} H\right), Z / H, \underline{\mathrm{id}}_{M}\right]\right)$ is an $F$-action isomorphic to $M \triangle \bar{\triangle} H$.

Projecting of the whole automorphism group along a normal quotient makes normal quotients special. An interesting observation made by Tucker [24] is that any $F$-action morphism $(\phi, \psi): M \rightarrow N$ factors through a normal quotient $M \rightarrow M \triangle \operatorname{ker} \psi \rightarrow N$, since $\mathrm{S}_{F}(M) \leq \mathrm{S}_{F}(M) \cdot \operatorname{Core}_{F}\left(\mathrm{~S}_{F}(N)\right) \leq \mathrm{S}_{F}(N)$ (by Theorem 3.4, $\operatorname{Core}_{F}\left(\mathrm{~S}_{F}(N)\right) \triangleleft F$ corresponds to ker $\left.\psi \triangleleft G_{M}\right)$.

\section{Parallel product and parallel-product decomposability}

The parallel product of two $F$-actions $M$ and $N$ is defined by

$$
M \| N=\left(\left(f_{M}, f_{N}\right), G, Z,\left(\underline{\mathrm{id}}_{M}, \underline{\mathrm{id}}_{N}\right)\right),
$$

where $G=\left(f_{M}, f_{N}\right)(F) \leq G_{M} \times G_{N}$ and $Z$ is the orbit of the induced action of $G$ on $Z_{1} \times Z_{2}$ which contains $\left(\underline{\mathrm{id}}_{M}, \underline{\mathrm{id}}_{N}\right)$. Since $\left(z_{1}, z_{2}\right) \cdot\left(g_{1}, g_{2}\right)=\left(z_{1}, z_{2}\right)$ if and only if $g_{1} \in\left(G_{M}\right)_{\underline{\mathrm{id}}_{M}}$ and $g_{2} \in\left(G_{N}\right)_{\underline{\mathrm{id}}_{N}}$, the kernel of the action is the direct product of the kernels of $\left(Z_{M}, G_{M}\right)$ and $\left(Z_{N}, G_{N}\right)$ and therefore trivial. The action $(Z, G)$ is faithful and transitive on $Z, M \| N$ is an $F$-action and $\mathrm{S}_{F}(M \| N)=\mathrm{S}_{F}(M) \cap \mathrm{S}_{F}(N)$. From the definition it follows that the parallel product is an associative and commutative operation (see also $[5,29])$. The definition enables us to construct $\operatorname{Mon}(M \| N)$ from $\operatorname{Mon}(M)$ and $\operatorname{Mon}(N)$ which is useful for computational purposes.

For examples on use of the parallel product, see [29].

Proposition 4.1 Let $M$ and $N$ be F-actions that both contain $\alpha_{w}$. Then $M \| N$ contains $\alpha_{w}$.

Proof $N_{F}\left(\mathrm{~S}_{F}(M)\right) \cap N_{F}\left(\mathrm{~S}_{F}(N)\right)$ is a subgroup of $N_{F}\left(\mathrm{~S}_{F}(M) \cap \mathrm{S}_{F}(N)\right)$.

The next proposition describes the relation between automorphisms, re-rootings and parallel products of re-rootings of an $F$-action $M$ through monodromy groups.

Proposition 4.2 Let $M$ be an $F$-action. Then for each $w \in F$,

1. $\operatorname{Mon}(M) \simeq \operatorname{Mon}\left(R_{w}(M)\right) \simeq \operatorname{Mon}\left(M \| R_{w}(M)\right)$.

2. $M \simeq R_{w}(M)$ if and only if $\alpha_{w} \in \operatorname{Aut}(M)$.

3. If $w^{2}=1$ then $\alpha_{w} \in \operatorname{Aut}\left(M \| R_{w}(M)\right)$.

4. Let $M^{M}$ denote the parallel product of all re-rootings of $M$. Then $M^{M}$ is regular and for any regular $F$-action $M^{\prime}$, such that $M \leq M^{\prime}$, it follows $M^{M} \leq M^{\prime}$.

Proof Let $K=\mathrm{S}_{F}(M), H=\operatorname{Core}_{F}(K)$ and $q: F \rightarrow F / H$ be the natural epimorphism. Since for any $w \in F, \mathrm{~S}_{F}\left(R_{w}(M)\right)=w^{-1} K w$ and $\operatorname{Core}_{F}\left(w^{-1} K w\right)=$ 
Core $_{F}\left(K \cap w^{-1} K w\right)=H$, all of the three monodromy groups in (1) are isomorphic to $(q, F / H)$ as $F$-groups. Since $\mathrm{S}_{F}\left(R_{w}(M)\right)=w^{-1} K w=K$ if and only if $w \in N_{F}(K)$, (2) follows. Since $w^{-1}\left(K \cap w^{-1} K w\right) w=w^{-1} K w \cap w^{-2} K w^{2}=$ $w^{-1} K w \cap K$ it follows $w \in N_{F}\left(K \cap w^{-1} K w\right)$ and (3) follows.

As $\mathrm{S}_{F}\left(M^{M}\right)=H, M^{M}$ is regular. For any $N \triangleleft F, N \leq w^{-1} K w$, for all $w \in F$, and it follows $N \leq H$. Hence, for any regular $F$-action $M^{\prime} \geq R_{w}(M)$, for all $w \in F$, it follows $M^{\prime} \geq \bar{M}^{M}$, yielding (4).

A consequence of the proposition is that for a regular $F$-action, the root can be ignored, since all re-rootings are isomorphic.

Proposition 4.3 Let $M$ and $N$ be $F$-actions and $f \in \operatorname{Aut}(F)$. Then $O_{f}(M \| N)=$ $O_{f}(M) \| O_{f}(N)$.

Proof The claim follows from the fact that $f^{-1}\left(\mathrm{~S}_{F}(M) \cap \mathrm{S}_{F}(N)\right)=f^{-1}\left(\mathrm{~S}_{F}(M)\right) \cap$ $f^{-1}\left(\mathrm{~S}_{F}(N)\right)$ as $f$ is an isomorphism.

A trivial $F$-action is a map $N$, such that $\mathrm{S}_{F}(N)=F$. A decomposition pair for an $F$-action $M$ is any pair of $F$-actions $\left(N_{1}, N_{2}\right)$, such that $M \simeq N_{1} \| N_{2}$ and neither of $N_{1}, N_{2}$ is isomorphic to $M$ or to a trivial map. This is equivalent to $\mathrm{S}_{F}(M) \lesseqgtr \mathrm{S}_{F}\left(N_{i}\right) \lesseqgtr F, i=1,2$. An $F$-action $M$ is parallel-product decomposable if there exists a decomposition pair for $M$. If there exists a decomposition pair consisting of normal quotients, then $M$ is normally parallel-product decomposable. If there exists a decomposition pair of strict $K$-quotients, then $M$ is strictly parallel-product decomposable.

Theorem 4.4 (Decomposition theorem) An F-action $M=(f, G, Z$, id) is parallelproduct decomposable if and only if there exist two different subgroups $K_{1}, K_{2} \leq G$, such that $G_{\underline{\mathrm{id}}} \lesseqgtr K_{i} \lesseqgtr G, i=1,2$, and $G_{\underline{\mathrm{id}}}=K_{1} \cap K_{2}$. Furthermore, $M$ is normally parallel-product decomposable if and only if there exist two different non-trivial normal subgroups $H_{1}, H_{2} \triangleleft G$ acting non-transitively on $Z$ and $G_{\underline{\mathrm{id}}} H_{1} \cap G_{\underline{\mathrm{id}}} H_{2}=G_{\underline{\mathrm{id}}}$. Also, $M$ is normally parallel-product decomposable if and only if it is strictly parallel-product decomposable.

Proof Consider the RTA morphism $(\mathrm{Id}, f):(Z, F, \underline{\mathrm{id}}) \rightarrow(Z, G, \underline{\mathrm{id}})$ in the $F$-action $M$. Then $\mathrm{S}_{F}(M)=f^{-1}\left(G_{\underline{\text { id }}}\right)=F_{\text {id }}$. By Theorem 3.4, such $K_{1}, K_{2}$ exist if and only if there exist $L_{i}, i=1,2$, where $F_{\text {id }} \lesseqgtr L_{i} \lesseqgtr F, f\left(L_{i}\right)=K_{i}, L_{i}=f^{-1}\left(K_{i}\right)$, and $L_{1} \cap L_{2}=F_{\text {id }}$. By Theorem 3.5, this is true if and only if $M$ is parallel-product decomposable and one of decomposition pairs is $\left(M / K_{1}, M / K_{2}\right)$.

Since $(Z, G)$ is faithful and transitive, $\operatorname{Core}_{G}\left(G_{\text {id }}\right)=\{1\}$ and non-triviality and non-transitivity of $H_{1}$ and $H_{2}$ is equivalent to $G_{\text {id }} \lesseqgtr G_{\text {id }} H_{i} \lesseqgtr G$. Together with the condition $G_{\underline{\text { id }}} H_{1} \cap G_{\underline{\text { id }}} H_{2}=G_{\text {id }}$ this is equivalent to normal parallel-product decomposability, where one of decomposition pairs is $\left(M / G_{\underline{\mathrm{id}}} H_{1}, M / G_{\mathrm{id}} H_{2}\right)$.

A map is strictly parallel-product decomposable if and only if there is a decomposition pair $\left(M / K_{1}, M / K_{2}\right)$, where the cores $N_{i}=\operatorname{Core}\left(K_{i}\right), i=1,2$, are nontrivial. But since $\operatorname{Core}_{G}\left(G_{\text {id }}\right)$ is trivial, $G_{\underline{\text { id }}} \lesseqgtr G_{\text {id }} N_{i} \leq K_{i}, i=1,2$, and obviously $G_{\underline{\text { id }}} N_{1} \cap G_{\underline{\text { id }}} N_{2}=G_{\underline{\text { id }}}\left(\right.$ as $\bar{K}_{1} \cap K_{2}=G_{\underline{\text { id }}}$ and $\left.G_{\underline{\text { id }}} \leq G_{\underline{\text { id }}} N_{1} \cap G_{\underline{\text { id }}} N_{2}\right)$. 
Fig. 1 Normal quotients of a 4-cycle on the sphere that yield a non-trivial normal parallel-product decomposition

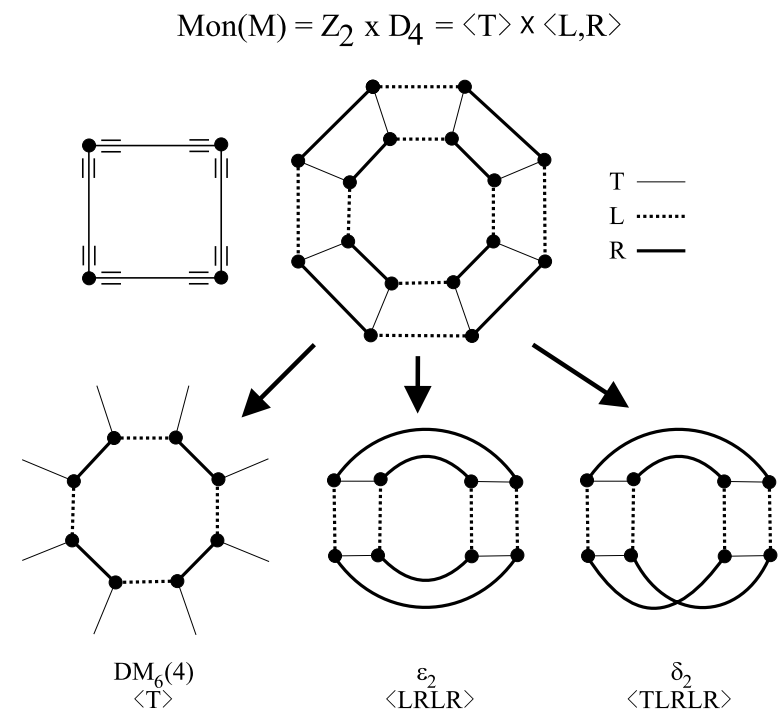

When computing with $F$-actions we mostly operate with (permutation) monodromy groups. The theorem tells us exactly how to determine decomposability of a map and how to decompose it, if possible. Often, we would like to decompose a monodromy group into a parallel product of monodromy groups of strictly smaller orders, i.e., we want strict parallel-product decomposability. The theorem says that if we are able to achieve this for a map $M$, we can do this in a way where both factors preserve the symmetry of $M$.

Let $M=(f, G, Z$, $\underline{\text { id }})$ be an $F$-action. There exist two normal non-trivial and non-transitive subgroups $H_{1}, H_{2} \triangleleft G$, such that $G_{\text {id }} H_{1} \cap G_{\text {id }} H_{2}=G_{\text {id }}$ if and only if there exist two minimal normal non-trivial and non-transitive subgroups $N_{1}, N_{2} \triangleleft G$, $N_{i} \leq H_{i}, i=1,2$, with $G_{\text {id }} N_{1} \cap G_{\text {id }} N_{2}=G_{\underline{\text { id }}}$. Therefore, it is sufficient to check minimal normal subgroups of $G$ to determine normal (or strict) parallel-product decomposability. Together with the fact that in a regular $F$-action the stabilizer in the monodromy group is trivial, the following theorem holds.

Theorem 4.5 A regular $F$-action $M$ is normally parallel-product decomposable if and only if $\operatorname{Mon}(M)$ (and thus also $\operatorname{Aut}(M)$ ) contains at least two non-trivial minimal normal subgroups. In this case both of the factors are regular $F$-actions.

Example 4.6 Figures 1 and 2 demonstrate an application of Theorem 4.5 and the difference between a normal and a general map quotient, both on the map $M$, a 4-cycle on the sphere. The monodromy group of $M$ is isomorphic to $\mathbb{Z}_{2} \times \mathrm{D}_{4}$ and has exactly 3 minimal normal subgroups which induce three normal quotients. In Fig. 1, $M$ and the three normal quotients are represented by flag graphs. By Theorem 4.5, $M$ is isomorphic to a parallel product of any two of the quotients.

In Fig. 2, a non-normal quotient $N$ is presented. Still, $N \| R_{L}(N) \simeq M$. Both of $N$ and $R_{L}(N)$ contain $\alpha_{T}$ and $\alpha_{R}$ which lift to $N \| R_{L}(N)$, by Proposition 4.1. Also, by Proposition 4.2, $\alpha_{L}$ lifts. Since $T, L$ and $R$ generate $F, N \| R_{L}(N)$ is reflexible. 

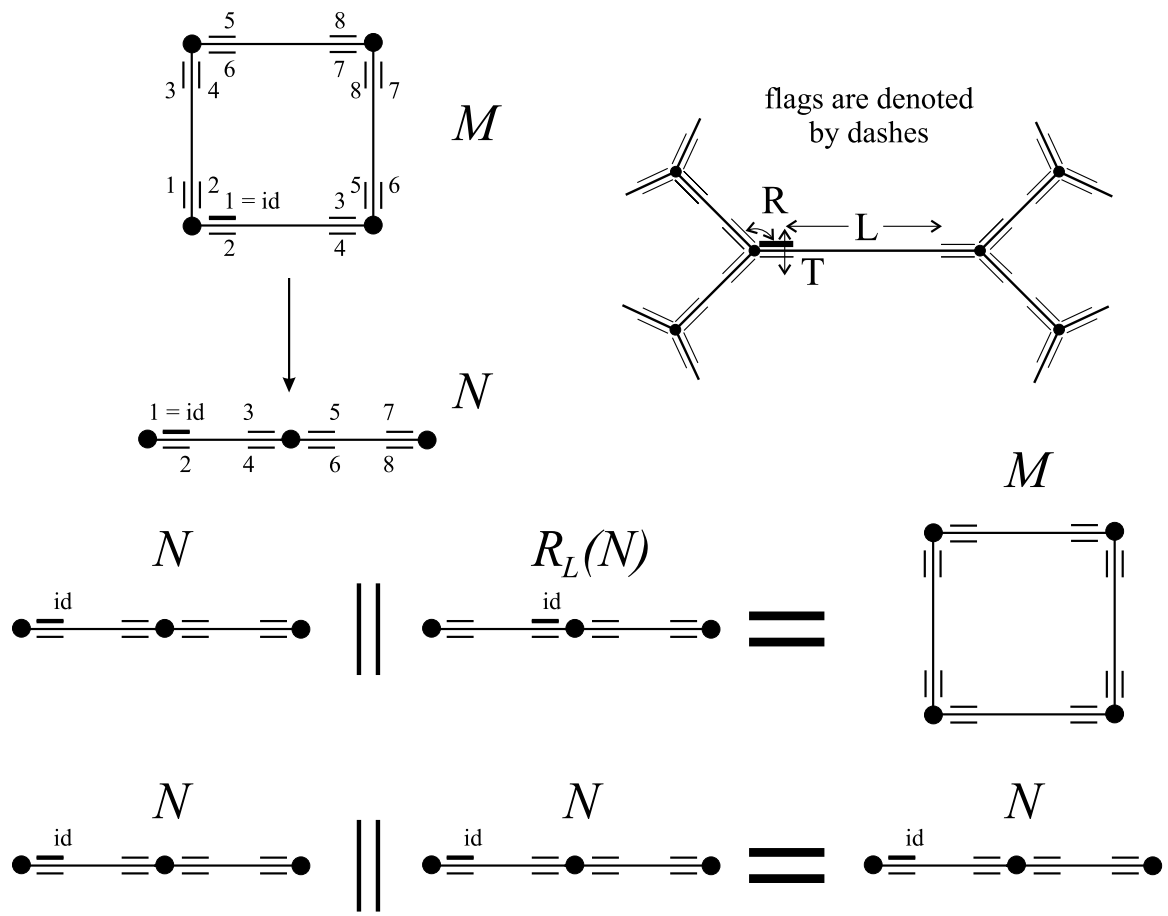

Fig. 2 A non-normal quotient of $C_{4}$

Consider a regular $F$-action $M$ and the $F$-action $\mathrm{M}_{\mathrm{F}}\left(\mathrm{S}_{F}(M)\right)=(q, F / H, F /$ $H, H)$, for $H=\mathrm{S}_{F}(M) \triangleleft F$ and $q: F \rightarrow F / H$, the natural epimorphism. Recall that $F=\left\langle a_{1}, \ldots, a_{k} \mid R_{1}=\cdots=R_{n}\right\rangle$. Since $F / H$ is finite, $H$ can be expressed as the normal closure of a finite set of words $\left\{R_{n+j}\right\}_{j=1}^{m}$ and $F / H=\left\langle a_{1}, \ldots, a_{k}\right| R_{1}=$ $\left.\cdots=R_{n+m}\right\rangle$. Note that the presentation of $F / H$ completely encodes all the information about $M$ up to isomorphism. Therefore a regular $F$-action can be represented by a finite presentation of $F / H$, which will be called a map group. Such presentations will be used in the remainder of the paper.

Consider a regular $F$-action $M$ represented with a map group $M=\left\langle a_{1}, \ldots, a_{k}\right|$ $\left.W_{1}^{e_{1}}=\cdots=W_{s}^{e_{s}}=1\right\rangle$, where $e_{i}$ is the exact order of the word $W_{i}$ in $M, i=1, \ldots, s$. A sequence of words $\left(W_{i}\right)_{i=1}^{S}$ is called the context. In the given context, $M$ can be encoded by the vector $\left(e_{i}\right)_{i=1}^{s}$ and we will write $M=\left(e_{i}\right)_{i=1}^{s}$. Let some other regular $F$-action $M^{\prime}$ be presented in a different context $C^{\prime}$. The common context $C^{\prime \prime}$ is any context which contains exactly all the words from $C$ and $C^{\prime}$. The $F$-action $M$ (and similarly $M^{\prime}$ ) can be represented in $C^{\prime \prime}$ by calculating the orders of the words in $C^{\prime \prime}$ and replacing the initial relations in $M$ with the new ones. For a given context $C$ and a regular $F$-action $M$, we will say that $C$ is sufficient for $M$, if $M$ has a presentation in $C$.

Let $M$ and $N$ be regular $F$-actions and $w \in F$. Let $a$ denote the exact order of $f_{M}(w)$ and $b$ denote the exact order of $f_{N}(w)$. Then the exact order of $\left(f_{M}, f_{N}\right)(w)$ is obviously $\operatorname{lcm}(a, b)$. The following lemma is straightforward. 
Lemma 4.7 Let $M=\left(a_{i}\right)_{i=1}^{s}, N=\left(b_{i}\right)_{i=1}^{s}$ be two regular $F$-actions represented in a common context $\left(W_{i}\right)_{i=1}^{s}$. Suppose that the common context is sufficient for the map $M \| N$. Then $M \| N=\left(\operatorname{lcm}\left(a_{i}, b_{i}\right)\right)_{i=1}^{s}$.

With this lemma the part of the paper dealing with $F$-actions in general is concluded. From now on we will deal with reflexible maps only.

\section{Degeneracy of reflexible maps}

In this section reflexible maps are classified into three families according to their degeneracy. For a given reflexible map $M$, let $e_{1}, \ldots, e_{7}$ be the exact orders of the words $T, L, R, T L, T R, L R, T L R$, respectively. A map $M$ is slightly-degenerate if it satisfies $e_{i} \geq 2$, for all $i=1, \ldots, 7$, and at least one of $e_{5}, e_{6}, e_{7}$ equals to 2 . It is degenerate if at least one of $e_{i}, i=1, \ldots, 7$, equals to 1 . If a map is not degenerate or slightly-degenerate then it is non-degenerate. In this case $e_{i} \geq 3, i=5,6,7$.

Note that the set of the chosen words represents exactly the generators whose orders determine the map's properties, such as the degrees of the vertices, the codegrees of the faces and the sizes of the Petrie circuits.

Let $C=\left(W_{i}\right)_{i=1}^{7}=(T, L, R, T L, T R, L R, T L R)$ be the context and $\left(e_{i}\right)_{i=1}^{7}$ be a vector denoting a map for which $C$ is sufficient. In analysis we use triality. Note that the operations $\mathrm{D}$ and $\mathrm{P}$ permute the triple $\left(e_{1}, e_{2}, e_{4}\right)$ with the same permutation as the triple $\left(e_{5}, e_{6}, e_{7}\right)$. To describe the action of $\mathrm{D}$ and $\mathrm{P}$ on the indices $i=1, \ldots, 7$ of $e_{i}$, we can represent $\mathrm{D}$ as a permutation $(1,2)(5,6)$ and $\mathrm{P}$ as $(2,4)(6,7)$.

Proposition 5.1 All degenerate reflexible maps are shown in Table 1.

Proof First we prove that all the map groups in Table 1 are uniquely determined by the context $C$. For all the maps in the table except $\mathrm{DM}_{5}(k), \mathrm{DM}_{6}(k)$ and $\mathrm{EM}_{3}(k)$,

Table 1 Degenerate reflexible maps

\begin{tabular}{lllllllll}
\hline Name & $(T$, & $L$, & $R$, & $T L$, & $T R$, & $L R$, & $T L R)$ & $|F(M)|$ \\
\hline $\mathrm{DM}_{1}$ & $(1$, & 1, & 1, & 1, & 1, & 1, & $1)$ & 1 \\
$\mathrm{DM}_{2}$ & $(1$, & 1, & 2, & 1, & 2, & 2, & $2)$ & 2 \\
$\mathrm{DM}_{4}$ & $(2$, & 1, & 1, & 2, & 2, & 1, & $2)$ & 2 \\
$\mathrm{DM}_{3}$ & $(1$, & 2, & 1, & 2, & 1, & 2, & $2)$ & 2 \\
$\mathrm{DM}_{8}$ & $(2$, & 2, & 1, & 1, & 2, & 2, & $1)$ & 2 \\
$\mathrm{DM}_{5}(k), k>0$ & $(2$, & 1, & 2, & 2, & $k$, & 2, & $k)$ & $2 k$ \\
$\mathrm{DM}_{6}(k), k>0$ & $(1$, & 2, & 2, & 2, & 2, & $k$, & $k)$ & $2 k$ \\
$\mathrm{EM}_{3}(k), k>0$ & $(2$, & 2, & 2, & 1, & $k$, & $k$, & $2)$ & $2 k$ \\
$\mathrm{DM}_{7}$ & $(2$, & 2, & 1, & 2, & 2, & 2, & $2)$ & 4 \\
$\mathrm{~K}_{2}$ & $(2$, & 2, & 2, & 2, & 1, & 2, & $2)$ & 4 \\
$\varepsilon_{1}$ & $(2$, & 2, & 2, & 2, & 2, & 1, & $2)$ & 4 \\
$\delta_{1}$ & $(2$, & 2, & 2, & 2, & 2, & 2, & $1)$ & 4 \\
\hline
\end{tabular}


Table 2 A map group of each map in this table is obtained as $\langle T, L, R| T^{2}=L^{2}=R^{2}=(T L)^{2}=(R T)^{2}=$ $\cdots=1\rangle$, where instead of "..." one should put the additional relations. All slightly-degenerate reflexible maps can be constructed from the maps in this table by using the operations D and P. Note that $\varepsilon_{1}$ and $\delta_{1}$ are degenerate and so not included in this table

\begin{tabular}{llc}
\hline Name & Additional relations & Order \\
\hline$\varepsilon_{k}, k>0$ even & $(L R)^{k},(T L R)^{k}$ & $4 k$ \\
$\varepsilon_{k}, k>1$ odd & $(L R)^{k},(T L R)^{2 k}$ & $4 k$ \\
$\delta_{k}, k>0$ even & $T(L R)^{k}, T(T L R)^{k}$ & $4 k$ \\
$\delta_{k}, k>1$ odd & $(L R)^{2 k},(T L R)^{k}$ & $4 k$ \\
\hline
\end{tabular}

this is pretty obvious. By triality it is enough to check the group of $\mathrm{DM}_{5}(k)$. The relations here determine a dihedral group $\mathrm{D}_{2 k}$ generated by $a=T$ and $b=T R$ and $\mathrm{D}_{2 k}=\left\langle a, b \mid a^{2}=b^{k}=(a b)^{2}=1\right\rangle$. One can easily see that any quotient of $\mathrm{D}_{2 k}$ strictly decreases the orders of at least one of the (projected) generators.

Now we will make an analysis of what kind of degenerate maps can occur. Let $e_{1}=e_{2}=1$. Then $e_{4}=1$. If $e_{3}=1$ we get $\mathrm{DM}_{1}$. If $e_{3}=2$ then it must be $e_{5}=$ $e_{6}=e_{7}=2\left(\mathrm{DM}_{2}\right)$. Now, let $e_{1}=1$ and $e_{2}=2$. Since $e_{4}=1$ implies $e_{2}=e_{1}$, it must be $e_{4}=2$. If $e_{3}=1$ then it must be $e_{5}=1, e_{6}=e_{7}=2\left(\mathrm{DM}_{3}\right.$ and by triality $\mathrm{DM}_{4}$ and $\left.\mathrm{DM}_{8}\right)$. If $e_{3}=2$ then $e_{5}=2$ and $e_{6}=e_{7}=k \geq 1\left(\mathrm{DM}_{6}(k)\right.$ and by triality $\mathrm{DM}_{5}(k)$ and $\left.\mathrm{EM}_{3}(k)\right)$. By triality, all the possibilities where one of $e_{1}, e_{2}, e_{4}$ is 1 are exhausted. Assume $e_{1}=e_{2}=e_{4}=2$. If $e_{3}=1$ then $e_{5}=e_{6}=e_{7}=2\left(\mathrm{DM}_{7}\right)$. Let now $e_{3}=2$. Since a map has to be degenerate, one of $e_{5}, e_{6}, e_{7}$ must be equal to 1 . By triality we can assume $e_{5}=1$. Then it must be $e_{6}=e_{7}=2$, otherwise the orders $e_{1}, e_{2}$ collapse $\left(\mathrm{K}_{2}, \varepsilon_{1}, \delta_{1}\right)$. This exhausts all the possibilities for degenerate maps.

A similar analysis of degenerate maps was done in [16], but their definition of degeneracy is different from ours and uses an automorphism group. According to [16], a reflexible map $M$ is degenerate if one of the generators $x=\alpha_{L}, y=\alpha_{T}$, $z=\alpha_{R} \in \operatorname{Aut}(M)$ equals to the identity. It is easy to see that their degeneracy is equivalent to saying that one of $e_{1}, e_{2}$ or $e_{3}$ is equal to 1 . (Note that the list in [16] omits the map $\mathrm{DM}_{8}$.)

In Fig. 3 all the flag graphs for degenerate maps are shown.

If a reflexible map is not degenerate then all the involutions $T, L, R, T L$ are fixedpoint-free. Such a map corresponds to a reflexible 2-cell embedding of some graph into a compact closed surface. Slightly-degenerate maps can be constructed using the operations D and $\mathrm{P}$ from a reflexible embedding of a cycle in some compact closed surface. The only possible such 2-cell embeddings are the embeddings of $k$-cycle in the sphere, denoted by $\varepsilon_{k}$, and in the projective plane with the $k$-cycle embedded as a non-contractible curve, denoted by $\delta_{k}$. Here the names are adopted from [28].

The map group presentations of maps $\varepsilon_{k}$ and $\delta_{k}$ are shown in Table 2.

\section{Normal parallel-product decomposition of reflexible maps}

Proposition 6.1 The map $\mathrm{DM}_{5}(k)\left(\mathrm{DM}_{6}(k), \mathrm{EM}_{3}(k)\right), k>2$ is normally parallelproduct decomposable if and only if $k$ is not a prime power. 


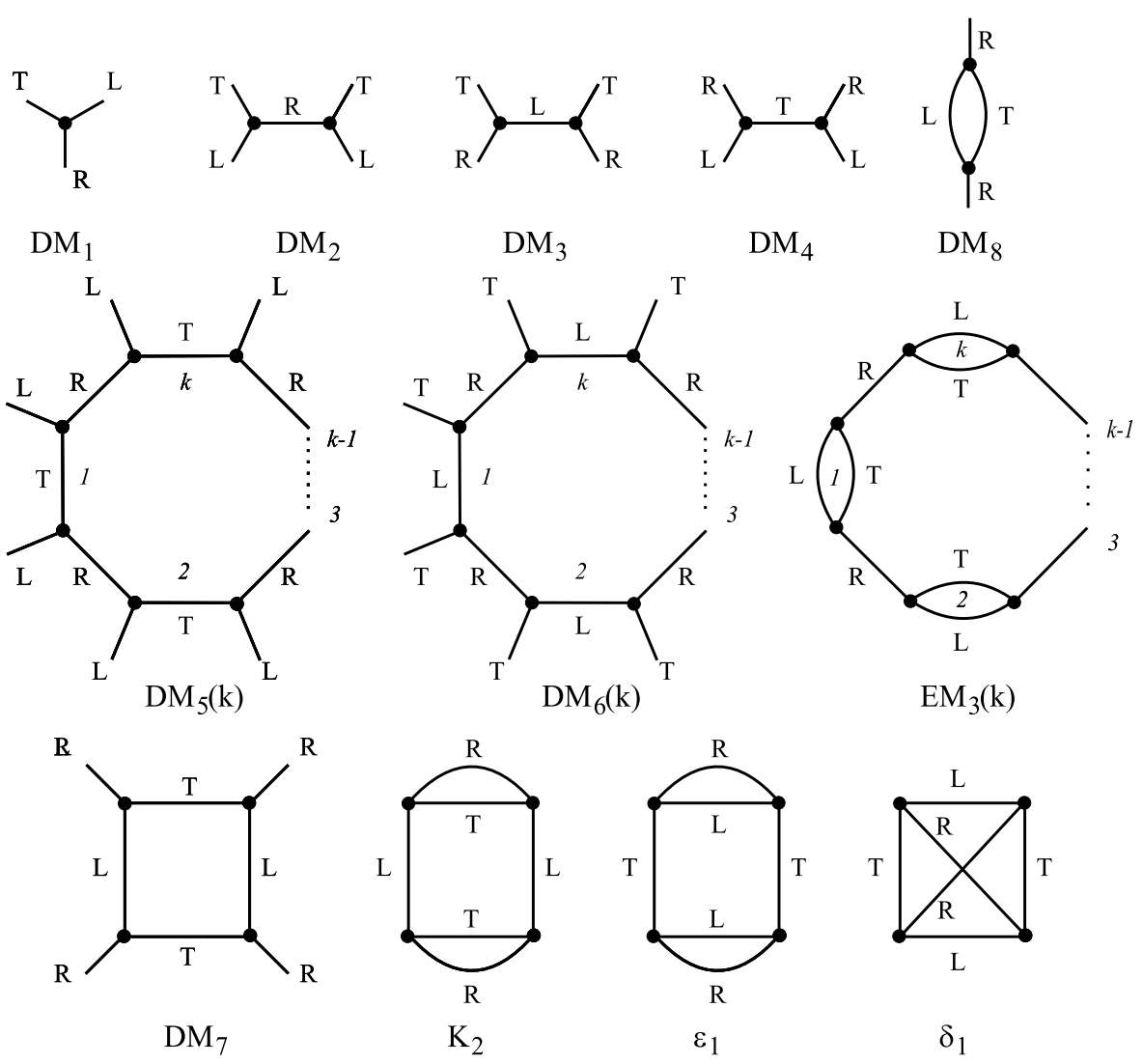

Fig. 3 Flag graphs of degenerate reflexible maps

Proof An integer $k$ is not a prime power if and only if there exist $a, b>1$, such that $\operatorname{gcd}(a, b)=1$ and $k=a b$. Using Lemma 4.7 and Table 1 it is easy to see that for any $a, b>1, \mathrm{DM}_{5}(a) \| \mathrm{DM}_{5}(b) \simeq \mathrm{DM}_{5}(\operatorname{lcm}(a, b))$. Nontrivial factors of $\mathrm{DM}_{5}(k)$ can be only degenerate maps with $L=1$, so only: $\mathrm{DM}_{5}(l), l \geq 1, \mathrm{DM}_{2}$ and $\mathrm{DM}_{4}$. Since $\mathrm{DM}_{2}$ and $\mathrm{DM}_{4}$ are quotients of any $\mathrm{DM}_{5}(l), l>2$, a parallel product with $\mathrm{DM}_{5}(l)$ absorbs them. Also $\mathrm{DM}_{2}\left\|\mathrm{DM}_{4} \simeq \mathrm{DM}_{2}\right\| \mathrm{DM}_{5}(1) \simeq \mathrm{DM}_{4} \| \mathrm{DM}_{5}(1) \simeq \mathrm{DM}_{5}(2)$. So if $k>2$ and $\mathrm{DM}_{5}(k)$ is normally parallel-product decomposable, then it must be a product of two factors of the form $\operatorname{DM}_{5}(l)$. By Table 1 and Lemma 4.7 this is possible only when the conditions of this proposition are fulfilled. Using triality, the proofs for $\mathrm{DM}_{6}(k)$ and $\mathrm{EM}_{3}(k)$ immediately follow.

The monodromy groups of the maps $\mathrm{DM}_{7}, \mathrm{~K}_{2}, \varepsilon_{1}$ and $\delta_{1}$, are isomorphic to $\mathbb{Z}_{2} \times \mathbb{Z}_{2}$ and thus by Theorem 4.5 the maps are normally parallel-product decomposable. The monodromy groups of $\mathrm{DM}_{1}, \mathrm{DM}_{2}, \mathrm{DM}_{3}, \mathrm{DM}_{4}$ and $\mathrm{DM}_{8}$ are either trivial or isomorphic to $\mathbb{Z}_{2}$, implying that those maps are normally parallel-product indecomposable.

The following corollary immediately follows. 
Corollary 6.2 All degenerate reflexible maps are normally parallel-product indecomposable except:

1. $\mathrm{DM}_{5}(k), \mathrm{DM}_{6}(k)$ and $\mathrm{EM}_{3}(k)$, for $k=2$ and any $k>2$ which is not a power of a prime,

2. $\mathrm{DM}_{7}, \mathrm{~K}_{2}, \varepsilon_{1}$ and $\delta_{1}$.

Note that $\mathrm{K}_{2}$ is a parallel product of any two maps in $\left\{\mathrm{DM}_{3}, \mathrm{DM}_{5}(1), \mathrm{EM}_{3}(1)\right\}$, $\mathrm{DM}_{7}$ of any two in $\left\{\mathrm{DM}_{3}, \mathrm{DM}_{4}, \mathrm{DM}_{8}\right\}, \varepsilon_{1}$ of any two in $\left\{\mathrm{DM}_{4}, \mathrm{DM}_{6}(1), \mathrm{EM}_{3}(1)\right\}$, $\delta_{1}$ of any two in $\left\{\mathrm{DM}_{5}(1), \mathrm{DM}_{6}(1), \mathrm{DM}_{8}\right\}$ and $\mathrm{DM}_{5}(2)$ of any two in $\left\{\mathrm{DM}_{2}, \mathrm{DM}_{4}\right.$, $\left.\mathrm{DM}_{5}(1)\right\}$.

Proposition 6.3 The only normally parallel-product indecomposable slightly-degenerate maps are the maps $\delta_{k}$, where $k=2^{n}, n \geq 1$.

Proof Since $\mathrm{P}\left(\varepsilon_{k}\right) \simeq \delta_{k}$, for $k$ odd, we have to consider only the normal parallelproduct decompositions of maps $\varepsilon_{k}$ for all $k>1$ and $\delta_{k}$, for $k>1$ even.

By Proposition 4.7, it is easily seen that $\varepsilon_{k} \simeq \mathrm{DM}_{6}(k) \| \mathrm{DM}_{4}$, for any $k>1$.

Now, let $k>0$ and let $l \geq 1$ be any odd number. The following map groups are defined by relations:

$$
\begin{aligned}
& F\left(\delta_{2^{k}}\right): T^{2}=L^{2}=R^{2}=(T L)^{2}=(R T)^{2}=1,(R L)^{2^{k}}=(T L R)^{2^{k}}=T \\
& F\left(\mathrm{DM}_{6}\left(2^{k} l\right)\right): T=L^{2}=R^{2}=(T L)^{2}=(R T)^{2}=(R L)^{2^{k} l}=(T L R)^{2^{k} l}=1 .
\end{aligned}
$$

A pretty straightforward relation chasing helps us to see that $\delta_{2^{k} l} \simeq \mathrm{DM}_{6}\left(2^{k} l\right) \| \delta_{2^{k}}$. This means that for any even $u$ not equal to the power of $2, \delta_{u}$ is normally parallelproduct decomposable.

For a given map $M$, denote by $e_{5}(M), e_{6}(M)$ and $e_{7}(M)$ the exponents of the words $R T, R L, T L R$, respectively. For $\delta_{2^{n}}, e_{5}=2, e_{6}=e_{7}=2^{n+1}$. Since these values are powers of 2 and $\operatorname{lcm}\left(2^{x}, 2^{y}\right)=\max \left(2^{x}, 2^{y}\right)$, at least one of $e_{5}, e_{6}, e_{7}$ must be reached with the corresponding values $e_{5}^{\prime}, e_{6}^{\prime}, e_{7}^{\prime}$ and $e_{5}^{\prime \prime}, e_{6}^{\prime \prime}, e_{7}^{\prime \prime}$ in two possible factors. Since the factors must be either degenerate or slightly degenerate maps, one of them must be one of $\mathrm{DM}_{6}\left(2^{n+1}\right), \delta_{2^{n}}$ or $\varepsilon_{2^{n+1}}$. A map $\delta_{2^{n}}$ is not admissible factor in a nontrivial decomposition, while a map $\varepsilon_{2^{n+1}}$ in a product would yield an orientable map (see [29]). Thus one of the factors must be $\mathrm{DM}_{6}\left(2^{n+1}\right)$. Since the context $C$ is not sufficient to obtain the map $\delta_{2^{n}}$, one of the maps must be $\delta_{l}$, for some $l=2^{u}, u<n$. But since $\operatorname{DM}_{6}\left(2^{n+1}\right) \| \delta_{l} \simeq \varepsilon_{2^{n+1}}$ this is not possible. Thus $\delta_{2^{n}}, n \geq 1$ is normally parallel-product indecomposable.

Using computer programs LowX [7] and MAGMA [3] all non-degenerate reflexible maps were calculated up to 100 edges. The results of the calculation match with Wilson's census of rotary maps [31]. Among them, the ones with the monolithic map group (i.e. having a unique minimal normal subgroup) were selected and they are shown in Table 3.

Claim 6.4 Up to triality, all normally parallel-product indecomposable nondegenerate reflexible maps up to 100 edges are presented in Table 3. 
Table 3 Normally parallel-product indecomposable non-degenerate reflexible maps up to triality and up to 100 edges. The second column is a reference to Wilson census [31]. A triple $e, s-f, n$, denotes that the corresponding map has the code $(e, n)$ in Wilson census, where $e$ denotes the number of edges. The map represents the triality class on maps with codes $(e, s),(e, s+1), \ldots,(e, f)$. A presentation of any of the maps in the table can be obtained by using a presentation $\langle T, L, R| T^{2}=L^{2}=R^{2}=(T L)^{2}=(R T)^{e_{5}}=$ $\left.(R L)^{e_{6}}=(T L R)^{e_{7}}=\cdots=1\right\rangle$, where the corresponding additional relations should be put instead of "...". The last column (Monolith) describes the minimal normal subgroups of the monodromy groups

\begin{tabular}{|c|c|c|c|c|c|c|}
\hline Name & Wilson cen. & $e_{5}$ & $e_{6}$ & $e_{7}$ & Additional relations & Monolith \\
\hline $\mathrm{MN}_{1}$ & $6,1-3,1$ & 3 & 3 & 4 & & $\mathbb{Z}_{2}^{2}$ \\
\hline $\mathrm{MN}_{2}$ & $8,2-4,2$ & 4 & 8 & 8 & $(R T R L)^{2},(L R T)^{2}(L R)^{2}$ & $\mathbb{Z}_{2}^{2}$ \\
\hline $\mathrm{MN}_{3}$ & $15,1-3,2$ & 3 & 5 & 5 & & $\{1\} \leq A_{5}$ \\
\hline $\mathrm{MN}_{4}$ & $16,1-1,1$ & 4 & 4 & 4 & & $\mathbb{Z}_{2}$ \\
\hline $\mathrm{MN}_{5}$ & $16,5-7,5$ & 4 & 8 & 8 & $\left((L R)^{2} T\right)^{2}$ & $\mathbb{Z}_{2}$ \\
\hline $\mathrm{MN}_{6}$ & $16,2-4,2$ & 4 & 16 & 16 & $(R T R L)^{2}, T L R T(L R)^{7}$ & $\mathbb{Z}_{2}$ \\
\hline $\mathrm{MN}_{7}$ & $18,1-3,1$ & 4 & 4 & 6 & $(R T R L)^{3}$ & $\mathbb{Z}_{3}^{\overline{2}}$ \\
\hline $\mathrm{MN}_{8}$ & $24,19-24,21$ & 3 & 8 & 12 & $(L R T L R)^{2} T(L R)^{2} T$ & $\mathbb{Z}_{2}$ \\
\hline $\mathrm{MN}_{9}$ & $24,28-33,30$ & 6 & 8 & 12 & $T L(R T)^{2}(L R)^{3}$ & $\mathbb{Z}_{2}$ \\
\hline $\mathrm{MN}_{10}$ & $27,1-3,3$ & 3 & 6 & 6 & & $\mathbb{Z}_{3}$ \\
\hline $\mathrm{MN}_{11}$ & $30,28-33,30$ & 4 & 5 & 6 & $L(R T R L)^{2}(R T)^{2}$ & $A_{5} \leq S_{5}$ \\
\hline $\mathrm{MN}_{12}$ & $30,37-37,37$ & 6 & 6 & 6 & $L(R T)^{2} R L(R T)^{3}, T(L R)^{3} T R(L R)^{2}$ & $A_{5} \leq S_{5}$ \\
\hline $\mathrm{MN}_{13}$ & $32,4-6,4$ & 4 & 4 & 8 & $(R T R L)^{4}$ & $\mathbb{Z}_{2}$ \\
\hline $\mathrm{MN}_{14}$ & $32,19-21,19$ & 4 & 16 & 16 & $(L R T)^{2}(R L)^{2}(R T)^{2},(L R)^{2} T(L R)^{6} T$ & $\mathbb{Z}_{2}^{2}$ \\
\hline $\mathrm{MN}_{15}$ & $32,1-3,1$ & 4 & 32 & 32 & $(R T R L)^{2},(L R T)^{2}(L R)^{14}$ & $\mathbb{Z}_{2}^{2}$ \\
\hline $\mathrm{MN}_{16}$ & $32,26-26,26$ & 8 & 8 & 8 & $(L R T)^{2}(R L)^{2}(R T)^{2},(L R T)^{2}(L R)^{2}(T R)^{2}$ & $\mathbb{Z}_{2}^{2}$ \\
\hline $\mathrm{MN}_{17}$ & $32,13-18,16$ & 8 & 16 & 16 & $(R T R L)^{2},(L R T)^{4}(L R)^{4}$ & $\mathbb{Z}_{2}$ \\
\hline $\mathrm{MN}_{18}$ & $40,19-21,21$ & 4 & 5 & 5 & & $\mathbb{Z}_{2}^{4}$ \\
\hline $\mathrm{MN}_{19}$ & $48,19-24,21$ & 3 & 6 & 8 & & $\mathbb{Z}_{2}^{2}$ \\
\hline $\mathrm{MN}_{20}$ & $48,70-72,72$ & 4 & 6 & 6 & $\left(T(L R)^{2}\right)^{3}$ & $\mathbb{Z}_{2}^{2}$ \\
\hline $\mathrm{MN}_{21}$ & $48,76-78,78$ & 6 & 6 & 8 & $\operatorname{LRTRLRTLRL}(R T)^{2}$ & $\mathbb{Z}_{2}^{2}$ \\
\hline $\mathrm{MN}_{22}$ & $48,73-75,75$ & 8 & 12 & 12 & $(R T)^{2}(L R)^{2} T R L R T L,(L R)^{3} T L(R L)^{2} R T$ & $\mathbb{Z}_{2}$ \\
\hline $\mathrm{MN}_{23}$ & $48,61-63,63$ & 8 & 24 & 24 & $\left(R T(R L)^{2}\right)^{2},(T L R)^{3}(L R)^{3}$ & $\mathbb{Z}_{2}$ \\
\hline $\mathrm{MN}_{24}$ & $48,64-66,66$ & 8 & 24 & 24 & $\left(R T(R L)^{2}\right)^{2}, T(L R)^{2} T(L R)^{2} L T R L R$ & $\mathbb{Z}_{2}$ \\
\hline $\mathrm{MN}_{25}$ & $50,1-3,1$ & 4 & 4 & 10 & $(R T R L)^{5}$ & $\mathbb{Z}_{5}^{2}$ \\
\hline $\mathrm{MN}_{26}$ & $54,10-15,13$ & 4 & 6 & 12 & $T(L R T R)^{3}$ & $\mathbb{Z}_{3}$ \\
\hline $\mathrm{MN}_{27}$ & $54,19-21,21$ & 6 & 12 & 12 & $L(R T)^{2} R L(R T)^{3},(T L R L R)^{3}$ & $\mathbb{Z}_{3}$ \\
\hline $\mathrm{MN}_{28}$ & $64,4-6,4$ & 4 & 4 & 8 & & $\mathbb{Z}_{2}$ \\
\hline $\mathrm{MN}_{29}$ & $64,49-54,51$ & 4 & 8 & 8 & $(L R T R)^{2}(L R)^{2} L T R L R T$ & $\mathbb{Z}_{2}^{2}$ \\
\hline $\mathrm{MN}_{30}$ & $64,40-42,42$ & 4 & 16 & 16 & $(R T R L)^{4},\left(R T R L(R L)^{2}\right)^{2},(L R T)^{4}(L R)^{4}$ & $\mathbb{Z}_{2}^{2}$ \\
\hline $\mathrm{MN}_{31}$ & $64,25-27,25$ & 4 & 32 & 32 & $(L R T)^{2}(R L)^{2}(R T)^{2},(L R)^{2} T(L R)^{14} T$ & $\mathbb{Z}_{2}^{2}$ \\
\hline $\mathrm{MN}_{32}$ & $64,1-3,1$ & 4 & 64 & 64 & $(R T R L)^{2}, T L R T(L R)^{31}$ & $\mathbb{Z}_{2}$ \\
\hline $\mathrm{MN}_{33}$ & $64,58-60,59$ & 8 & 8 & 8 & $(L R T)^{2}(L R)^{2}(T R)^{2}, T(R T R L) T(R T R L)^{3}$ & $\mathbb{Z}_{2}^{2}$ \\
\hline $\mathrm{MN}_{34}$ & $64,34-36,34$ & 8 & 16 & 16 & $(L R T R L R T)^{2},(R T)^{2} R L(R T)^{2}(R L)^{3}$ & $\mathbb{Z}_{2}$ \\
\hline $\mathrm{MN}_{35}$ & $64,43-45,45$ & 8 & 16 & 16 & $\begin{array}{l}(L R T)^{2}(R L)^{2}(R T)^{2},\left((R T)^{3} R L\right)^{2} \\
(L R T)^{2}(L R)^{2} T(L R)^{3} L T R\end{array}$ & $\mathbb{Z}_{2}$ \\
\hline $\mathrm{MN}_{36}$ & $64,7-9,7$ & 8 & 16 & 16 & $\left((L R)^{2} T\right)^{2}$ & $\mathbb{Z}_{2}$ \\
\hline $\mathrm{MN}_{37}$ & $64,19-24,24$ & 8 & 32 & 32 & $(R T R L)^{2},(L R T)^{4}(L R)^{12}$ & $\mathbb{Z}_{2}$ \\
\hline $\mathrm{MN}_{38}$ & $75,7-12,9$ & 3 & 6 & 10 & & $\mathbb{Z}_{5}^{2}$ \\
\hline $\mathrm{MN}_{39}$ & $80,37-39,39$ & 5 & 5 & 8 & $(L R T R)^{2} T(L R)^{2} T R L R T$ & $\mathbb{Z}_{2}$ \\
\hline $\mathrm{MN}_{40}$ & $80,40-45,42$ & 5 & 8 & 10 & $\left(R T(R L)^{3}\right)^{2},(T L R)^{3} T R(L R)^{2} T R$ & $\mathbb{Z}_{2}$ \\
\hline $\mathrm{MN}_{41}$ & $80,46-48,46$ & 8 & 10 & 10 & $(R T)^{3}(L R)^{4} T L,(T L R)^{3} L R(T R)^{2} L R$ & $\mathbb{Z}_{2}$ \\
\hline $\mathrm{MN}_{42}$ & $81,1-6,3$ & 3 & 6 & 18 & $\left((L R)^{2} T\right)^{6}$ & $\mathbb{Z}_{3}$ \\
\hline $\mathrm{MN}_{43}$ & $81,28-33,31$ & 6 & 6 & 9 & $(L R T L R)^{2} T(L R)^{2} T, T\left(L R(T R)^{2}\right)^{3}$ & $\mathbb{Z}_{3}$ \\
\hline $\mathrm{MN}_{44}$ & $81,22-27,27$ & 6 & 9 & 18 & $\left(R T(R L)^{2}\right)^{2},(L R T)^{4} R L(R T)^{2}$ & $\mathbb{Z}_{3}$ \\
\hline $\mathrm{MN}_{45}$ & $84,28-33,30$ & 3 & 7 & 8 & & $\operatorname{PSL}(2,7)$ \\
\hline $\mathrm{MN}_{46}$ & $84,49-51,49$ & 3 & 8 & 8 & $(T L R)^{2}(L R T)^{2}(L R)^{3} L T(R L)^{2} R$ & $\operatorname{PSL}(2,7)$ \\
\hline $\mathrm{MN}_{47}$ & $84,43-48,44$ & 4 & 6 & 8 & $T(R T R L)^{4},\left(R T(R L)^{2}\right)^{3}$ & $\operatorname{PSL}(2,7)$ \\
\hline $\mathrm{MN}_{48}$ & $84,37-42,39$ & 4 & 7 & 8 & $(R T R L)^{3}$ & $\operatorname{PSL}(2,7)$ \\
\hline $\mathrm{MN}_{49}$ & $84,53-55,55$ & 6 & 6 & 8 & $\left(L(T R)^{2}\right)^{3},\left(T(L R)^{2}\right)^{3}$ & $\operatorname{PSL}(2,7)$ \\
\hline $\mathrm{MN}_{50}$ & $84,34-36,34$ & 6 & 7 & 7 & $R T L(R T)^{2} R L(R T)^{2}$ & $\operatorname{PSL}(2,7)$ \\
\hline $\mathrm{MN}_{51}$ & $84,52-52,52$ & 8 & 8 & 8 & $\begin{array}{l}(R T R L)^{3}, T L(R T)^{2} L R T R L(T R)^{2}, \\
\left(T(L R)^{2}\right)^{3}\end{array}$ & $\operatorname{PSL}(2,7)$ \\
\hline $\mathrm{MN}_{52}$ & $96,82-87,85$ & 4 & 6 & 24 & $(L R T)^{3}(R L)^{2} T R L(R T)^{2}$ & $\mathbb{Z}_{2}$ \\
\hline $\mathrm{MN}_{53}$ & $96,73-78,76$ & 4 & 12 & 24 & $(L R T L R)^{2} L T R L R T$ & $\mathbb{Z}_{2}$ \\
\hline
\end{tabular}


Table 3 (Continued)

\begin{tabular}{|c|c|c|c|c|c|c|}
\hline Name & Wilson cen. & $e_{5}$ & $e_{6}$ & $e_{7}$ & Additional relations & Monolith \\
\hline $\mathrm{MN}_{54}$ & $96,184-186,186$ & 6 & 6 & 8 & $(R T R L)^{3}, L(R T)^{2}(L R)^{2} L(T R)^{2} T(L R)^{2} T$ & $\mathbb{Z}_{2}$ \\
\hline $\mathrm{MN}_{55}$ & $96,187-189,189$ & 6 & 6 & 8 & $(L R T)^{3}(R T R L)^{2} R$ & $\mathbb{Z}_{2}^{2}$ \\
\hline $\mathrm{MN}_{56}$ & $96,178-180,180$ & 8 & 12 & 12 & $\begin{array}{l}\left(T(L R)^{2}\right)^{3},\left((R T)^{3} R L\right)^{2} \\
(R T R L)^{4}, L(R T)^{3}(L R)^{5} T\end{array}$ & $\mathbb{Z}_{2}$ \\
\hline $\mathrm{MN}_{57}$ & $96,181-183,183$ & 8 & 12 & 12 & $\begin{array}{l}\left((R T)^{3} R L\right)^{2},(R T R L)^{4}, \\
T(L R)^{2} T(R L)^{3} R T R L R, L(R T)^{3}(L R)^{5} T\end{array}$ & $\mathbb{Z}_{2}$ \\
\hline $\mathrm{MN}_{58}$ & $96,97-99,99$ & 8 & 24 & 24 & $\begin{array}{l}L(R T)^{2}(L R)^{2} T R L R T \\
\left((L R)^{3} T\right)^{2}(L R)^{6}\end{array}$ & $\mathbb{Z}_{2}$ \\
\hline $\mathrm{MN}_{59}$ & $96,64-69,68$ & 8 & 48 & 48 & $\begin{array}{l}\left(R T(R L)^{2}\right)^{2},(L R T)^{2} R T L R L T(R T)^{2}, \\
(T L R)^{3}(L R)^{9}\end{array}$ & $\mathbb{Z}_{2}$ \\
\hline $\mathrm{MN}_{60}$ & $98,1-3,1$ & 4 & 4 & 14 & $(R T R L)^{7}$ & $\mathbb{Z}_{7}^{2}$ \\
\hline
\end{tabular}

Table 4 The normally parallel-product indecomposable non-degenerate reflexible maps $\mathrm{MN}_{1}$ to $\mathrm{MN}_{10}$ in detail. A genus symbol contains genera of the maps $M, \mathrm{P}(M)$ and $\operatorname{PDP}(M)$. Note that the operation $\mathrm{D}$ preserves the genus of a map while the operation $\mathrm{P}$ preserves its underlying graph. An entry $x \geq 0$ in a genus symbol denotes orientable genus $x$, while $x<0$ denotes nonorientable genus $-x$. The hexagonal number is the number of nonisomorphic maps in the triality class. Underlying graphs of maps $M, \mathrm{D}(M)$ and $\operatorname{PDP}(M)$ are described in the last column. An edge multiplicity $k>1$ of an underlying graph $X$ is denoted by $X(k)$

\begin{tabular}{llll}
\hline Name & Genus symbol & Hex. n. & Underlying graphs \\
\hline $\mathrm{MN}_{1}$ & {$[0,-1,-1]$} & 3 & $K_{4}, K_{4}, C_{3}(2)$ \\
$\mathrm{MN}_{2}$ & {$[2,2,3]$} & 3 & $C_{4}(2), K_{2}(8), K_{2}(8)$ \\
$\mathrm{MN}_{3}$ & {$[-1,-1,-5]$} & 3 & ${\text { Petersen, } K_{6}, K_{6}}$ \\
$\mathrm{MN}_{4}$ & {$[1,1,1]$} & 1 & $K_{4,4}, K_{4,4}, K_{4,4}$ \\
$\mathrm{MN}_{5}$ & {$[3,3,5]$} & 3 & $K_{4,4}, C_{4}(4), C_{4}(4)$ \\
$\mathrm{MN}_{6}$ & {$[4,4,7]$} & 3 & $C_{8}(2), K_{2}(16), K_{2}(16)$ \\
$\mathrm{MN}_{7}$ & {$[1,-5,-5]$} & 3 & $D K_{3,3,3}, D K_{3,3,3}, K_{3,3}(2)$ \\
$\mathrm{MN}_{8}$ & {$[2,3,-16]$} & 6 & Gen. Petersen $G(8,3), K_{2,2,2}(2), K_{4}(4)$ \\
$\mathrm{MN}_{9}$ & {$[6,7,-16]$} & 6 & $Q_{3}(2), K_{2,2,2}(2), K_{4}(4)$ \\
$\mathrm{MN}_{10}$ & {$[1,1,-11]$} & 3 & Pappus, $K_{3,3,3}, K_{3,3,3}$ \\
\hline
\end{tabular}

There are exactly 2424 reflexible maps up to 100 edges. Among them, there are 1223 non-degenerate and they are presented in [31]; 229 of non-degenerate are normally parallel-product indecomposable and are obtained from Table 3 (calculating whole triality classes). There are 1201 degenerate and slightly degenerate maps, among which 203 are normally parallel-product indecomposable and are obtained from the classification above.

As an example of an application of the results in this paper, Table 5 provides some decompositions of representatives of triality classes for all reflexible normally parallel-product decomposable maps in Wilson census up to 20 edges. Note that for each map in the table there are in general several other possible decompositions (some of them might also have normally parallel-product decomposable factors).

\section{Conclusion}

The main results of the paper are establishing the theory of $F$-actions, a characterization of $F$-action morphisms through $K$-quotients, the decomposition theorem and its application to the classification of reflexible maps of at most 100 edges. The most important conclusion of the paper is that the classification of reflexible maps 
Table 5 Some decompositions of representatives of the first 10 decomposable triality classes from Wilson census [31]. A triple $e, s-f, n$, denotes that the corresponding map has the code $(e, n)$ in Wilson census, where $e$ denotes the number of edges. The map represents the triality class on maps with codes $(e, s)$, $(e, s+1), \ldots,(e, f)$

\begin{tabular}{llllll}
\hline Wilson cen. & $e_{5}$ & $e_{6}$ & $e_{7}$ & Name & Some decompositions \\
\hline $8,1-1,1$ & 4 & 4 & 4 & $(4,4) 2,0$ & $\mathrm{DM}_{6}(4)\left\|\mathrm{EM}_{3}(4), \delta_{2}\right\| D\left(\delta_{2}\right), \mathrm{EM}_{3}(4)\left\|\mathrm{DM}_{5}(4), \mathrm{DM}_{6}(4)\right\| \mathrm{DM}_{5}(4)$ \\
$9,1-3,2$ & 3 & 6 & 6 & $B^{*}(3,6)$ & $\mathrm{EM}_{3}(3) \| \mathrm{DM}_{5}(3)$ \\
$12,1-6,1$ & 3 & 4 & 6 & $\mathrm{Cube}$ & $\mathrm{EM}_{3}(1)\left\|P\left(\mathrm{MN}_{1}\right), P\left(\mathrm{MN}_{1}\right)\right\| \mathrm{DM}_{3}$ \\
$12,7-12,7$ & 4 & 6 & 12 & $(6,4 \mid 2)$ & $\mathrm{DM}_{6}(3)\left\|\mathrm{DM}_{5}(4), \mathrm{DM}_{6}(3)\right\| D\left(\delta_{2}\right)$ \\
$12,13-15,15$ & 4 & 6 & 6 & $D\left(\Gamma_{2}\right)$ & $\mathrm{DM}_{2}\left\|D P\left(\mathrm{MN}_{1}\right), D P\left(\mathrm{MN}_{1}\right)\right\| \mathrm{DM}_{3}$ \\
$15,4-9,5$ & 6 & 10 & 15 & $P\left(M^{\prime}(15,4)\right)$ & $\mathrm{DM}_{6}(5) \| \mathrm{DM}_{5}(3)$ \\
$16,8-10,10$ & 4 & 8 & 8 & $B(4,8,3,0)$ & $\mathrm{EM}_{3}(4)\left\|\mathrm{MN}_{2}, \mathrm{MN}_{2}\right\| \delta_{4}, \mathrm{DM}_{6}(8)\left\|\mathrm{DM}_{5}(4), \delta_{4}\right\| D\left(\delta_{2}\right)$ \\
$18,4-6,5$ & 6 & 6 & 6 & $D(B(6,6))$ & $\mathrm{EM}_{3}(3)\left\|\mathrm{DM}_{2}\right\| \mathrm{DM}_{6}(3), \mathrm{EM}_{3}(3)\left\|\mathrm{DM}_{5}(1)\right\| \mathrm{DM}_{6}(3)$ \\
$18,7-9,9$ & 4 & 9 & 9 & $D\left(\Gamma_{3}\right)$ & $D P\left(\mathrm{MN}_{1}\right) \| \mathrm{DM}_{6}(9)$ \\
$20,1-6,2$ & 4 & 10 & 20 & $P\left(M^{\prime}(20,9)\right)$ & $\mathrm{DM}_{6}(5)\left\|D\left(\delta_{2}\right), \mathrm{DM}_{6}(5)\right\| \mathrm{DM}_{5}(4)$ \\
\hline
\end{tabular}

can be reduced to monolithic groups generated with three involutions, two of which commute. In $[19,20]$ the application of the theory of this paper is extended to edgetransitive maps. Similarly it can be extended to orientably regular maps, hypermaps and abstract polytopes.

Acknowledgement I would like to express my gratitude to my supervisors Tomaž Pisanski and Thomas W. Tucker and to Dragan Marušič for their guidance and support. I would also like to thank Dušanka Janežič and the National Chemical Institute in Ljubljana for letting me use their computer cluster Vrana [14]. Also, the author acknowledges the extensive use of programs LowX [8] and MAGMA [3]. I thank the referees for many valuable suggestions.

\section{References}

1. Archdeacon, D., Gvozdjak, P., \& Širán, J. (1997). Constructing and forbidding automorphisms in lifted maps. Mathematica Slovaca, 47(2), 113-129.

2. Bergau, P., \& Garbe, D. (1989). Non-orientable and orientable regular maps. In Proceedings of groups-Korea 1998, Lecture notes in mathematics (Vol. 1398, pp. 29-42). New York: Springer.

3. Bosma, W., Cannon, C., \& Playoust, C. (1997). The Magma algebra system I: the user language. Journal of Symbolic Computation, 24, 235-265.

4. Brahana, H. R. (1927). Regular maps and their groups. American Journal of Mathematics, 49, 268284.

5. Breda d'Azevedo, A., \& Nedela, R. (2001). Join and intersection of hypermaps. Acta Universitatis M. Belii Series Mathematics, 9, 13-28.

6. Conder, M. http://www.math.auckland.ac.nz/ conder/.

7. Conder, M., \& Dobcsányi, P. (2001). Determination of all regular maps of small genus. Journal of Combinatorial Theory Series B, 81, 224-242.

8. Conder, M., \& Dobcsányi, P. (1999). Computer program LowX, censuses of rotary maps. http://www. math.auckland.ac.nz/ peter.

9. Coxeter, H. S. M., \& Moser, W. O. J. (1984). Generators and relations for discrete groups (4th ed.). Berlin: Springer.

10. Ferri, M. (1976). Una rappresentazione delle $n$-varieta topologiche triangolabili mediante grafi $(n+1)$-colorati. Bollettino Unione Matematica Italiana Sezione B (5), 13(1), 250-260.

11. Garbe, D. (1969). Über die regulären Zerlegungen geschlossener orientierbarer Flächen. Journal für die Reine und Angewandte Mathematik, 237, 39-55.

12. Hartley, M. I. (1999). All polytopes are quotients, and isomorphic polytopes are quotients by conjugate subgroups. Discrete \& Computation Geometry, 21(2), 289-298.

13. Hartley, M. I. (1999). More on quotient polytopes. Aequationes Mathematicae, 57, 108-120.

14. Hodošček, M., Borštnik, U., \& Janežič, D. (2002). Crow for large scale macromolecular simulations. Cell. Mol. Biol. Letters, 7(1), 118-119. 
15. Jones, G. A., \& Thornton, J. S. (1983). Operations on maps, and outer automorphisms. Journal of Combinatorial Theory Series B, 35(2), 93-103.

16. Li, C. H., \& Širáň, J. (2005). Regular maps whose groups do not act faithfully on vertices, edges, or faces. European Journal of Combinatorics, 26(3-4), 521-541.

17. Lins, S. (1982). Graph-encoded maps. Journal of Combinatorial Theory Series B, 32(2), 171-181.

18. Malnič, A., Nedela, R., \& Škoviera, M. (2002). Regular homomorphisms and regular maps. European Journal of Combinatorics, 23, 449-461.

19. Orbanić, A. (2005). Parallel-product decomposition of edge-transitive maps. http://arxiv.org/PS_ cache/math/pdf/0510/0510428.pdf.

20. Orbanić, A. (2006). Edge-transitive maps. Doctoral dissertation, University of Ljubljana, Ljubljana.

21. Richter, R. B., Širáň, J., Jajcay, R., Tucker, T. W., \& Watkins, M. E. (2005). Cayley maps. Journal of Combinatorial Theory Series B, 95, 189-245.

22. Sherk, F. A. (1959). The regular maps on a surface of genus three. Canadian Journal of Mathematics, $11,452-480$.

23. Širáň, J., Tucker, T. W., \& Watkins, M. E. (2001). Realizing finite edge-transitive orientable maps. Journal of Graph Theory, 37, 1-34.

24. Tucker, T. W.: Private communication.

25. Vince, A. (1983). Combinatorial maps. Journal of Combinatorial Theory Series B, 34(1), 1-21.

26. Wilson, S. E. (1976). New techniques for the construction of regular maps. Doctoral dissertation, University of Washington, Seattle.

27. Wilson, S. E. (1978). Riemann surfaces over regular maps. Canadian Journal of Mathematics, 30(4), $763-782$.

28. Wilson, S. E. (1985). Bicontactual regular maps. Pacific Journal of Mathematics, 120(2), 437-451.

29. Wilson, S. E. (1994). Parallel products in groups and maps. Journal of Algebra, 167, 539-546.

30. Wilson, S. E. (2002). Families of regular graphs in regular maps. Journal of Combinatorial Theory Series B, 85(2), 269-289.

31. Wilson, S. E. (2005). Wilson's census of rotary maps. http://www.ijp.si/RegularMaps/.

32. Zvonkin, A. (2001). Megamaps: construction and examples. In Discrete mathematics and theoretical computer science proceedings (pp. 329-340). Paris: MIMD. 